\title{
Antioxidants in Wild Mushrooms
}

\author{
Isabel C.F.R. Ferreira*, Lillian Barros and Rui M.V. Abreu \\ CIMO-ESAB, Instituto Politécnico de Bragança, Campus de Sta. Apolónia, 1172, 5301-855 Bragança, Portugal
}

\begin{abstract}
Maintenance of equilibrium between free radical production and antioxidant defences (enzymatic and non enzymatic) is an essential condition for normal organism functioning. When this equilibrium has a tendency for the production of free radicals we say that the organism is in oxidative stress. In this situation, excess free radicals may damage cellular lipids, proteins and DNA, affecting normal function and leading to various diseases. In aerobic organisms, the free radicals are constantly produced during the normal cellular metabolism, mainly in the form of Reactive Oxygen Species (ROS) and Reactive Nitrogen Species (RNS). Exposition of the organism to free radicals has led to the development of endogenous defence mechanisms to eliminate them. These defences were the response of evolution to the inevitability of ROS production in aerobic conditions. Natural products with antioxidant activity may help the endogenous defence system. In this perspective the antioxidants present in the diet assume a major importance as possible protector agents reducing oxidative damage. Particularly, the antioxidant properties of wild mushrooms have been extensively studied by our research group and by others, and many antioxidant compounds extracted from these sources have been identified, such as phenolic compounds, tocopherols, ascorbic acid, and carotenoids. We will review the compounds identified so far in mushrooms, as well as the mechanism of action involved in their antioxidant properties. Wild mushrooms might be used directly in diet and promote health, taking advantage of the additive and synergistic effects of all the bioactive compounds present.
\end{abstract}

\section{OXIDATIVE STRESS}

Free radicals are produced in the normal natural metabolism of aerobic cells, mostly in the form of oxygen reactive species (ROS). Once produced, most of the free radicals are neutralized by cellular antioxidant defences (enzymes and non-enzymatic molecules). Maintenance of equilibrium between free radicals production and antioxidant defences is an essential condition for normal organism functioning [1].

Beneficial effects of ROS occur at low or moderate concentrations and involve cellular physiological roles of signalization and regulation [2,3]. Nevertheless, the equilibrium between ROS production and antioxidant defences might be displaced either by the overproduction of ROS or by the loss of the cell antioxidant defences [4]. This disequilibrium is known as oxidative stress, and in this case, the excess ROS may oxidize and damage cellular lipids, proteins and DNA, leading to their modification and inhibiting their normal function $[1,5,6]$.

Oxidative stress might have natural causes such as extreme exercise or inflammation processes, or non-natural causes such as the presence of xenobiotics in the organism or situations related to several diseases (Fig. 1). In fact, the noncontrolled production of free radicals has been related to more than one hundred diseases including several kinds of cancer [7], diabetes [1], cirrhoses [8], cardiovascular diseases [9], neurological disorders [10], among others [1]. The overproduction of ROS has also been related to the aging process [11-13].

Considering that $70 \%$ of the chronical diseases and related costs can be prevented, the knowledge about ROS and about their overproduction control is crucial [14]. This control can be achieved by the maintenance of good levels of antioxidants and free radicals scavengers, increasing diet quality (higher consumption of vegetables, leguminous and fruits) or avoiding behaviours that lead to a higher ROS production, such as tobacco, excessive exposure to environmental polluents and xenobiotics [15].

\section{FREE RADICALS, REACTIVE OXYGEN SPECIES (ROS) AND REACTIVE NITROGEN SPECIES (RNS)}

A free radical is defined as any atom or molecule possessing unpaired electrons in the outer orbit $[16,17]$. They are generally unstable and very reactive. Free radicals derived from molecular Oxygen $\left(\mathrm{O}_{2}\right)$ are usually known by reactive Oxygen species (ROS) and represent the most important class of radical species generated in living systems [18]. In fact, despite the importance of $\mathrm{O}_{2}$ to aerobic life, in some conditions it can be toxic. This phenomenon is called "oxygen paradox" [19]. Several ROS production pathways and the main endogenous antioxidant defences of the cell are described in Fig. (2).

The addition of one electron to molecular Oxygen forms the superoxide anion $\left(\mathrm{O}_{2}{ }^{-}\right)$, which is considered the "primary" ROS. Superoxide anion is mostly produced in mitochondria, due to a small but continuous "leak" of the electrons in the mitochondrial electron transport system (ETS). These electrons generate superoxide anion instead of reducing oxygen to water. Measurements on submitochondrial particles suggest an upper limit of $1-3 \%$ of "leaking" electrons in the mitochondrial ETS [20]. Superoxide anion can also be produced by different endogenous enzymatic systems present in the cell like NADPH oxidases and xanthine oxidase $[3,19] . \mathrm{O}_{2}^{-}$have been implicated in several diseases [21].

Even though $\mathrm{O}_{2}{ }^{-}$is not a very active radical, it can interact with other molecules generating what are considered as "secondary" ROS, such as hydrogen peroxide $\left(\mathrm{H}_{2} \mathrm{O}_{2}\right)$ and hydroxyl radical $\left(\mathrm{OH}^{*}\right)$. Hydroxyl radical has a very short life time but is considered to be the most toxic among all ROS, being responsible for the attack to DNA molecules, damaging purins and pyrimidines and the structure of desoxyribose DNA [22]. Hydroxyl radical is the neutral form 


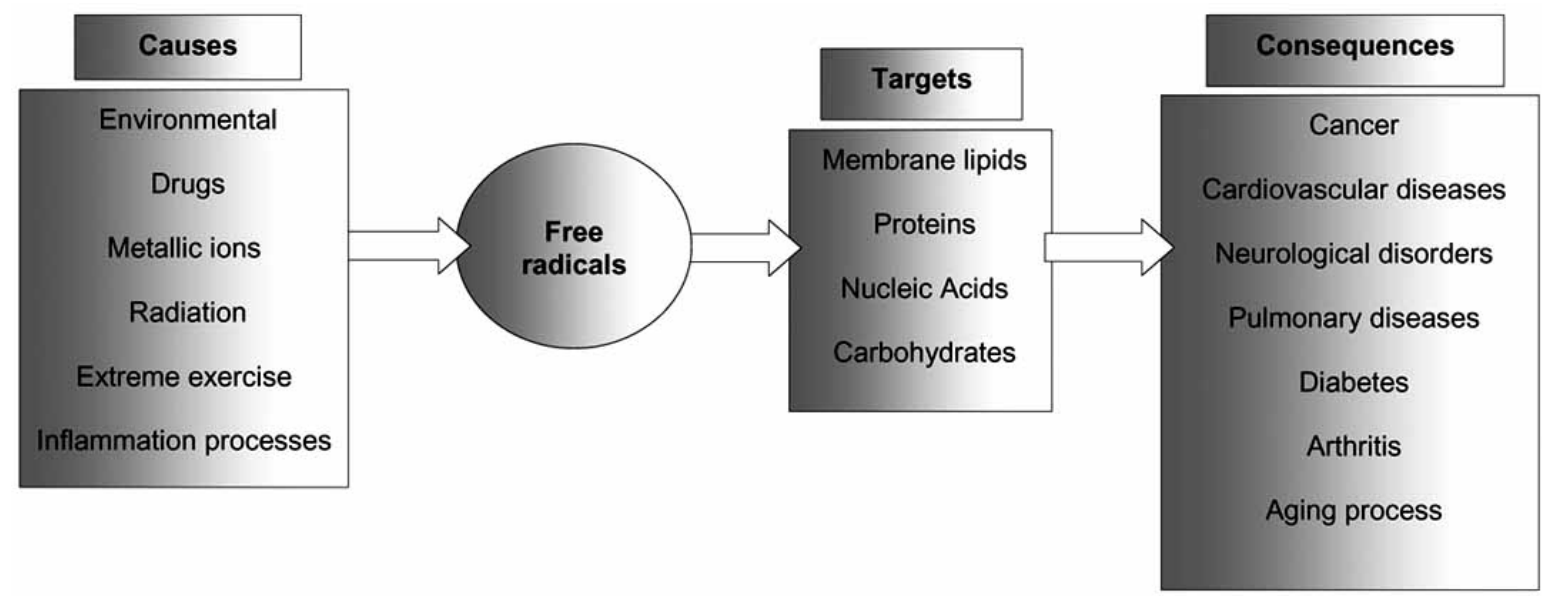

Fig. (1). Major causes for over production of free radical (oxidative stress), possible cellular targets and conditions that were associated to oxidative stress.

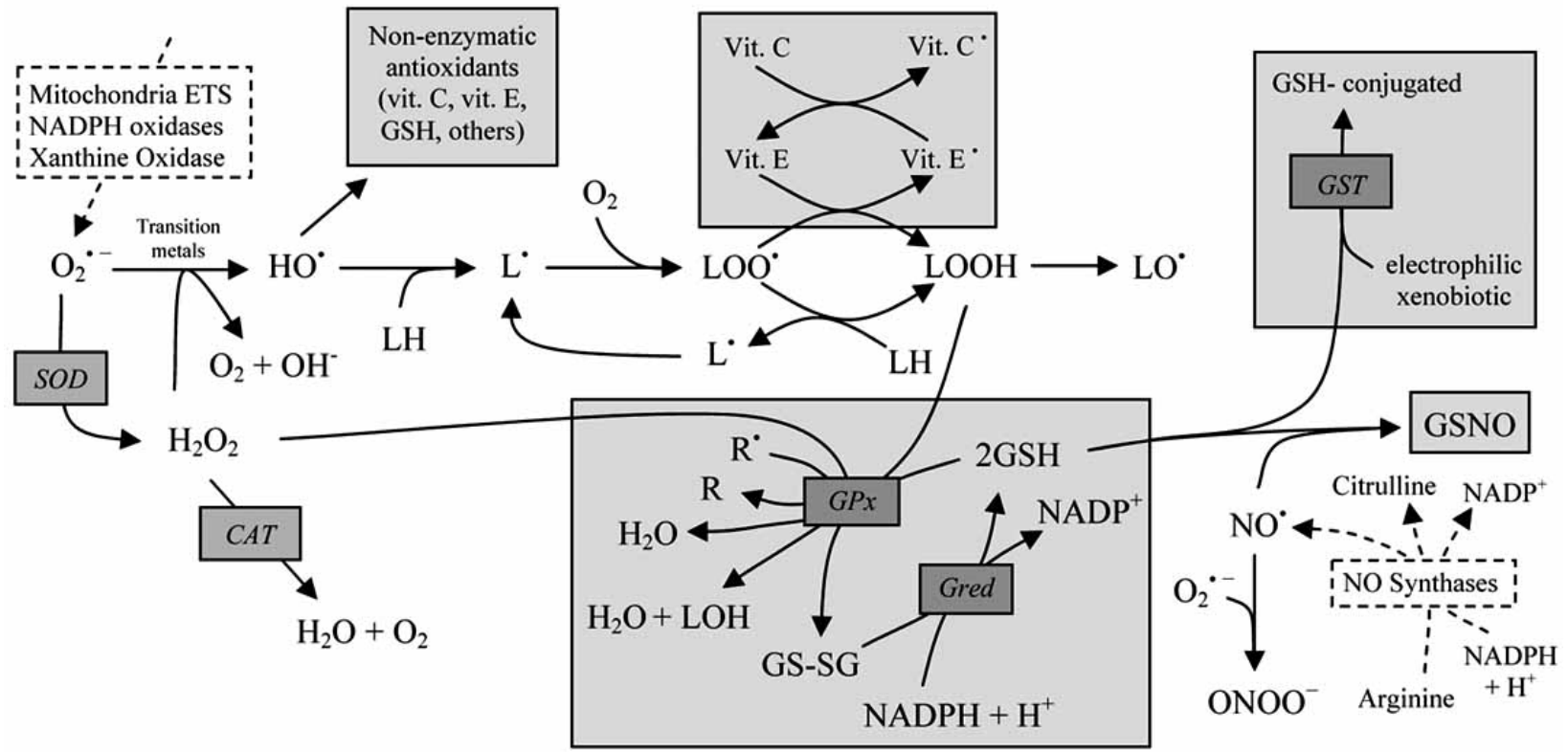

Fig. (2). Overview of the main reactions involving reactive Oxygen species (ROS) / reactive Nitrogen species (RNS), and major endogenous enzymatic and non-enzymatic antioxidant defences in the cell. The most representative endogenous sources (traced rectangles) of ROS/RNS are presented and include: Mitochondrial ETS (Electron transport system), NADPH oxidases, Xanthine oxidase for ROS and NO synthases for RNS. The main antioxidant defences are presented in shaded rectangles and the enzymes involved are presented in italic. Molecular Oxygen $\left(\mathrm{O}_{2}\right)$, superoxide anion $\left(\mathrm{O}_{2}{ }^{-}\right)$, hydrogen peroxide $\left(\mathrm{H}_{2} \mathrm{O}_{2}\right)$, hydroxyl radical $\left(\mathrm{HO}^{\circ}\right)$, hydroxide ion $\left(\mathrm{HO}^{-}\right)$membrane lipids $(\mathrm{LH})$, lipid radical $\left(\mathrm{L}^{\circ}\right)$, peroxyl radical $\left(\mathrm{LOO}^{\circ}\right)$, hydroperoxide lipid $(\mathrm{LOOH})$, lipid alkoxyl radical $\left(\mathrm{LO}^{\circ}\right)$, nitric oxide $\left(\mathrm{NO}^{\circ}\right)$, radicals $\left(\mathrm{R}^{*}\right)$, non-radicals $(\mathrm{R})$, alcohols (LOH), glutathione (GSH), glutathione disulphide (GS-SG), $\alpha$-tocopherol or vitamin E (vit. E), vitamin E radical (vit. E'), vitamin C (vit. C), vitamin $\mathrm{C}$ radical (vit. $\mathrm{C}^{*}$ ), S-nitrosoglutathione (GSNO), nicotidamide adenine dinucleotide phosphate: oxidized $\left(\mathrm{NADP}^{+}\right)$, reduced (NADPH). Enzymes: Superoxide dismutase (SOD), catalase (CAT), glutathione peroxidase (GPx), glutathione redutase (Gred), glutathioneS-transferases (GST), Mitochondrial ETS (electron transport system), nitric oxide synthase (NOS).

of the hydroxide ion and it is formed by an electron transfer from transition metals to $\mathrm{H}_{2} \mathrm{O}_{2}$, and interacts with biomolecules immediately after generation. Eventual permanent damages in the genetic material caused by oxidative stress might represent the first step to mutagenesis, carcinogenesis and aging [1].

Mitochondria are the most important source of ROS, but they are also the first targets of these radicals because ROS have an easy access to the membrane lipids, which are susceptible to free radicals attack. This attack is called lipid peroxidation and promotes the production of different types of ROS [23] (Fig. 2). The lipid peroxidation usually begins with the extraction of a hydrogen atom from a polyunsaturated lipid (LH) chain, through the action of reactive species such as $\mathrm{HO}^{\circ}$. This generates a highly reactive lipid radical $\left(\mathrm{L}^{\circ}\right)$ that can react with $\mathrm{O}_{2}$ to form a peroxyl radical ( $\mathrm{LOO}^{\circ}$ ). 
If not neutralized by antioxidants defences, the peroxyl radical will react with other adjacent lipids producing hydroperoxides lipids ( $\mathrm{LOOH})$ that can easily be decomposed to form new $\mathrm{L}^{\cdot}$ radicals, initiating a process that is known as chain propagation reactions. This process when not stopped, can lead to much superior damage than the ROS that started the reaction $[3,24,25]$.

It is also important to notice the existence of radicals with Nitrogen called reactive Nitrogen species (RNS). The principal RNS is nitric oxide $\left(\mathrm{NO}^{\circ}\right)$ and it is generated in biological tissues by specific nitric oxide synthases (NOS), which metabolise arginine to citrulline (Fig. 2) [3, 26]. NO is an abundant reactive radical that acts as an important oxidative biological signalling molecule in a large variety of physiological processes, including neurotransmission, blood pressure regulation, defence mechanisms, and regulation of immune response $[3,27]$. The over expression of RNS is called nitrosative stress and may lead to nitrosylation of proteins and so affect their normal function [6]. Cells of the immune system produce both the superoxide anion and nitric oxide during the oxidative burst triggered in inflammatory processes. Under these conditions, $\mathrm{NO}^{\circ}$ can react with $\mathrm{O}_{2}{ }^{-}$ to produce significant amounts of peroxynitrite anion $\left(\mathrm{ONOO}^{-}\right)$, which is a potent oxidising agent that can cause DNA fragmentation and lipid oxidation [28, 29].

\section{ENDOGENOUS ANTIOXIDANT DEFENCES}

Exposure to free radicals from a variety of sources has led organisms to the development of a series of defence mechanisms (Fig. 2) [20]. These defences were the evolution response to the inevitability of the existence of oxygen radicals in aerobic life conditions, and can be classified into enzymatic and non-enzymatic.

There are many different endogenous enzymatic antioxidant defences in the organism, either in intracellular or extracellular medium. Examples of these defences include superoxide dismutase (SOD), catalase (CAT), glutathione peroxidases (GPx), and glutathione redutase (Gred) among others. The endogenous non-enzymatic antioxidant defences include glutathione (GSH), $\alpha$-tocopherol (vitamin E), ascorbic acid (vitamin $\mathrm{C}$ ), lipoic acid, and other antioxidants [1, 2, 30]. SOD converts $\mathrm{O}_{2}{ }^{--}$into $\mathrm{H}_{2} \mathrm{O}_{2}$, which is then detoxified to water either by CAT in the peroxysomes or by GPx in the mitochondria, cytosol or nucleus. GPx are a group of selenoenzymes that require Selenium on their biosynthesis. The intake on food or dietary supplements of Selenium is crucial for antioxidant enzyme defences. This is of special importance as mushrooms have been found to contain Selenium in good quantity especially wild edible mushrooms [31]. Another important enzyme is Gred, which regenerates GSH that is used as a hydrogen donor by GPx [32]. GPx can also transform hydroperoxide lipids into alcohols (LOH).

Glutathione (GSH) is a low molecular weight tripeptide composed of glutamate, cysteine, and glycine being the main intracellular redox buffer. The capacity of GSH to regenerate the most important antioxidant molecules is linked with the redox state of the glutathione disulphide/glutathione (GSSG/GSH) couple [33]. GSH effectively scavenges ROS $\left(\mathrm{HO}^{\circ}, \mathrm{H}_{2} \mathrm{O}_{2}, \mathrm{LOO}^{\circ}\right.$ and $\left.\mathrm{ONOO}^{-}\right)$either directly or indirectly as a cofactor of several detoxifying enzymes, e.g. GPx, GST, among others. In the neutralization process of ROS, GSH is oxidized to glutathione disulphide (GS-SG), which can be further reduced to two GSH by the enzyme Gred. GSH is also able to regenerate other antioxidant molecules such as vitamins $\mathrm{C}$ and $\mathrm{E}$. GSH can also react with a variety of electrophilic xenobiotics in reactions catalysed by glutathione-Stransferases (GST) generating products with higher solubility and thus easier to eliminate. GSH can also neutralize $\mathrm{NO}^{\circ}$, resulting in the formation of S-nitrosoglutathione (GSNO) $[1,2]$.

Vitamin E is a liposoluble vitamin present in the membranes thus playing an important role in the prevention of lipid peroxidation. Among the eight forms of vitamin $\mathrm{E}, \alpha-$ tocopherol is the most active form in humans. ROS (hydroxyl and peroxyl radicals, etc.) react with vitamin $\mathrm{E}$, generating a poorly reactive phenolic radical (vit. $\mathrm{E}^{*}$ ). Vitamin $\mathrm{C}$ then reacts with vit. $\mathrm{E}^{\cdot}$ producing vitamin $\mathrm{C}$ radical (vit. $\mathrm{C}^{\circ}$ ) and regenerating vitamin $\mathrm{E}$. Both radicals (vit. $\mathrm{E}^{\circ}$ and vit. $\mathrm{C}^{\circ}$ ) are poorly reactive species because of its unpaired electron [2, 34].

Besides all the mentioned endogenous defences, antioxidant supplements or antioxidant-containing foods may be used to help the organism to reduce oxidative damage or to protect food quality by preventing oxidative deterioration [17]. In recent years, the restriction in the use of synthetic antioxidants, such as BHA (2-tert-butyl-4-methoxyphenol) and BHT (2,6-di-tert-butyl-4-methylphenol), has caused an increased interest towards natural antioxidant substances [35, 36]. Natural antioxidants are being extensively studied for their capacity to protect organisms and cells from damage brought on by oxidative stress.

A multitude of natural antioxidants have already been isolated from different kinds of plant materials such as oilseeds, cereal crops, vegetables, fruits, leaves, roots, spices, and herbs [37]. Epidemiological studies have consistently shown that a high dietary intake of fruits and vegetables is strongly associated with reduced risk of developing chronic diseases, such as cancer and cardiovascular disease [38-42]. In fact, the implication of oxidative and nitrosative stress in the etiology and progression of several acute and chronic clinical disorders has led to the suggestion that antioxidants can have health benefits as prophylactic agents [39]. This suggests that changes in dietary behaviour, increasing consumption of plant-based foods, which contain significant amounts of bioactive phytochemicals, may provide desirable health benefits, beyond basic nutrition, to reduce the risk of chronic diseases [43].

The number of individual phytochemicals already identified in fruits and vegetables is estimated in $>5,000$, but a large percentage still remains unknown and need to be identified before we can fully understand the health benefits of phytochemicals in whole foods [38].

\section{WILD MUSHROOMS AS A SOURCE OF ANTIOXI- DANTS}

Mushrooms have become attractive as functional foods and as a source of physiologically beneficial medicine [4447]. Some advantages of using mushrooms over plants as 
Table 1. Studies on Antioxidant Properties of Wild Mushrooms

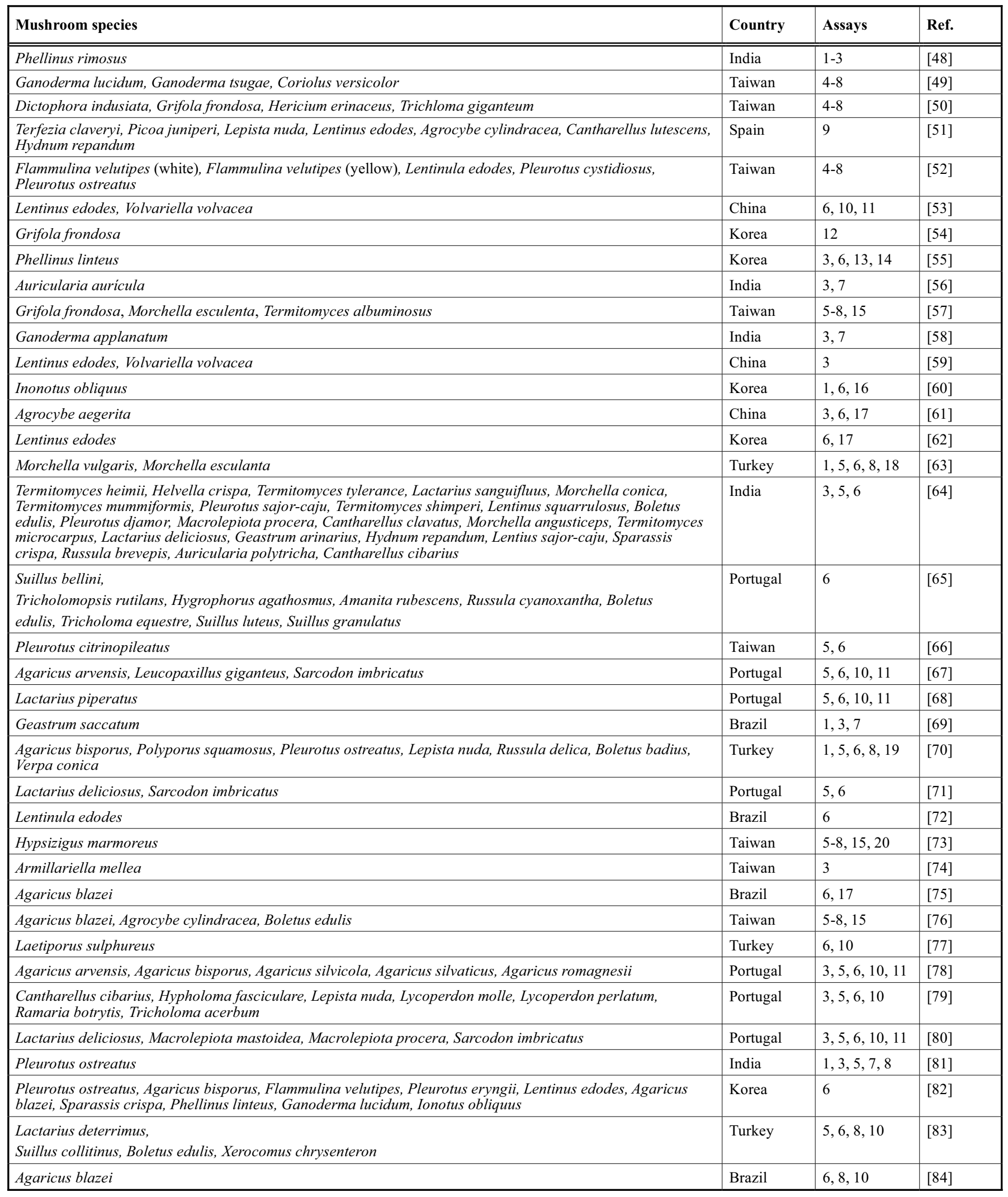

1- Superoxide anion radical scavenging activity; 2- Nitric oxide scavenging activity; 3- Thiobarbituric reactive substances (TBARS assay); 4- 1,3-diethyl-2-thiobarbituric acid (DETBA) method; 5- Reducing power; 6- 2,2-Diphenyl-1-picrylhydrazyl (DPPH) scavenging activity; 7- Hydroxyl radicals scavenging activity; 8- Chelating effects on ferrous ions; 9- Linoleic acid assay; 10- $\beta$-carotene bleaching inhibition; 11- Hemolysis inhibition; 12- SOD activity; 13- Xanthine oxidase inhibition; 14- Chorioallantoic membrane (CAM) assay; 15- Conjugated diene method; 16- 2,7-dichlorofluorescindiacetate (DCF)/2,2'-azobis(2-amidinopropane)dihydrochloride (AAPH) assay; 17- 2,2'-azinobis-(3-ethylbenzthiazoline-6sulphoronic acid $\left(\mathrm{ABTS}^{+}\right)$radical cation scavenging activity; 18- Hydrogen peroxide scavenging activity; 19- Thiocyanate method; 20- Chelating effects on cupric ions. 
sources of bioactive compounds are that often the fruiting body can be produced in much less time, the mycelium may also be rapidly produced in liquid culture and the culture medium can be manipulated to produce optimal quantities of active products.

Different wild mushroom species were reported to have antioxidant activity, which was mainly related to their phenolic content [48-84]. Several protocols were used to determine the mushrooms antioxidant activity (Table 1).

There are some works reporting the influence of processing conditions, growth conditions and growing stages of mushrooms, on the antioxidant potential. Our research group [80] described that the amount of antioxidants in cooked samples of Lactarius deliciosus, Macrolepiota mastoidea, Macrolepiota procera and Sarcodon imbricatus significantly decreased. We explained that heat used in the cooking procedure could destroy the structures of polyphenols and cause a decrease in their antioxidant activity. Nevertheless, at low heating temperatures, an increase in phenolics concentration may occur. This was observed by us in the dried mushrooms and by Choi et al. [62] who described that heat treatment of Lentinus edodes increased the overall content of free polyphenolic and flavonoid compounds. The authors suggested that heat treatment might produce changes in their extractability due to the disruption of the cell wall thus bound polyphenolic and flavonoid compounds may be released more easily relative to those of raw materials. Another reason for the improved antioxidant activity could be the formation of novel compounds having antioxidant activities during heat treatment or thermal processing. Valentão et al. [85] described that the conservation procedures such as preservation in olive oil or vinegar, seem to affect the qualitative and quantitative phenolics and organic acids profiles of Cantharellus cibarius. Particularly, ascorbic acid increased significantly in the samples preserved in vinegar. The mushrooms gowth stage also influences their antioxidant contents [86]. In fact, fruiting bodies of Lactarius deliciosus and Lactarius piperatus in a mature stage with mature spores, revealed lower content in antioxidants such as phenolics, ascorbic acid and $\beta$-carotene. This was explained with the involvement of those compounds in defence mechanisms inherent to the aging process (presence of mature spores), resulting in the lowering of their contents in the most advanced stage.

The antioxidants found in mushrooms are mainly phenolic compounds (phenolic acids and flavonoids), followed by tocopherols, ascorbic acid and carotenoids. These molecules were quantified in tens of different species mainly from Finland, India, Korea, Poland, Portugal, Taiwan and Turkey (Table 2). The values are avalialable in literature, but expressed in different basis (dry weight, fresh weight and extract). Helvella crispa from India revealed the highest content of phenolic compounds expressed per $\mathrm{g}$ of extract (34.65 $\mathrm{mg} / \mathrm{g}$ ), while Sparassis crispa from Korea revealed the highest value expressed in a dry weight basis $(0.76 \mathrm{mg} / \mathrm{g})$. Auricularia fuscosuccinea (white) from Taiwan $(32.46 \mathrm{mg} / \mathrm{g}$ of extract), Agaricus silvaticus $\left(3.23 \times 10^{-3} \mathrm{mg} / \mathrm{g}\right.$ of dry weight $)$ and Ramaria Botrytis $\left(2.50 \times 10^{-4} \mathrm{mg} / \mathrm{g}\right.$ of fresh weight) from Portugal, were the richest species in tocopherols. Auricularia fuscosuccinea (brown) from Taiwan
(11.24 mg/g of extract), Suillus collinitus from Portugal (3.79 mg/g of dry weight) and Agaricus bisporus from Poland $(0.22 \mathrm{mg} / \mathrm{g}$ of fresh weight) revealed the highest levels of ascorbic acid. Lactarius deliciosus from Portugal revealed the highest contents in $\beta$-carotene $(0.09 \mathrm{mg} / \mathrm{g}$ of extract).

\section{Phenolic Compounds}

There are a large number of manuscripts reporting determination of total phenolics by Folin Ciocalteu's assay [4884]. Phenolic compounds include different subclasses (flavonoids, phenolic acids, stilbenes, lignans, tannins, oxidized polyphenols) displaying a large diversity of structures, some of which may escape the usual methodologies of analysis, commonly carried out by HPLC (High Performance Liquid Chromatography) coupled to distinct detection devices. Various reasons exist for that, like the existence of isomers, difficulty for chromatographic separation of some compounds, lack of commercial standards, or structure not yet elucidated [96]. The method of Folin Ciocalteu's is, therefore, largely used to evaluate total phenolics despite all the interferences of this assay since the reagent (mixture of phosphotungstic acid and phosphomolibdic acid) also reacts with other non-phenolic reducing compounds leading to an overvaluation of the phenolic content. For instance, ascorbic acid is a widespread reducing agent that can interfere in the Folin-Ciocalteu's reaction [96]. Other reducing substances such as some sugars and amino acids could also interfere. In addition, the results have to be expressed in equivalents of a particular standard compound (like catechin, gallic acid or tannin acid). All these aspects increase the importance of the determination of phenolic compounds by more sophisticated techniques.

A few studies concerning the analysis of the phenolic components of wild mushrooms can be found in the literature $[64,65,81,82,85,88,89,92]$. The individual profiles of phenolic compounds were obtained by high-performance liquid chromatography coupled to photodiode array detector (HPLC-DAD) [64, 65, 82, 85, 88, 92], or to an ultraviolet detector [81], or by gas chromatography-mass spectrometry selected ion monitoring (GC-MS SIM) [89]. Table 3 presents the phenolic compounds found in several mushroom species.

Phenolic compounds are aromatic hydroxylated compounds, possessing one or more aromatic rings with one or more hydroxyl groups, being commonly found in vegetables, fruits and many food sources that form a significant portion of our diet, and some of which are among the most potent and therapeutically useful bioactive substances [97]. Natural phenolic compounds accumulate as end-products from the shikimate and acetate pathways and can range from relatively simple molecules (phenolic acids, phenylpropanoids, flavonoids) to highly polymerised compounds (lignins, melanins, tannins), with flavonoids representing the most common and widely distributed sub-group [98].

As described above, the main phenolic compounds found in mushrooms were phenolic acids. Phenolic acids can be divided into two major groups, hydroxybenzoic acids (Fig. 3) and hydroxycinnamic acids (Fig. 4), which are derived from non-phenolic molecules benzoic and cinnamic acid, respectively. 
Table 2. Antioxidants Quantified in Wild Mushrooms

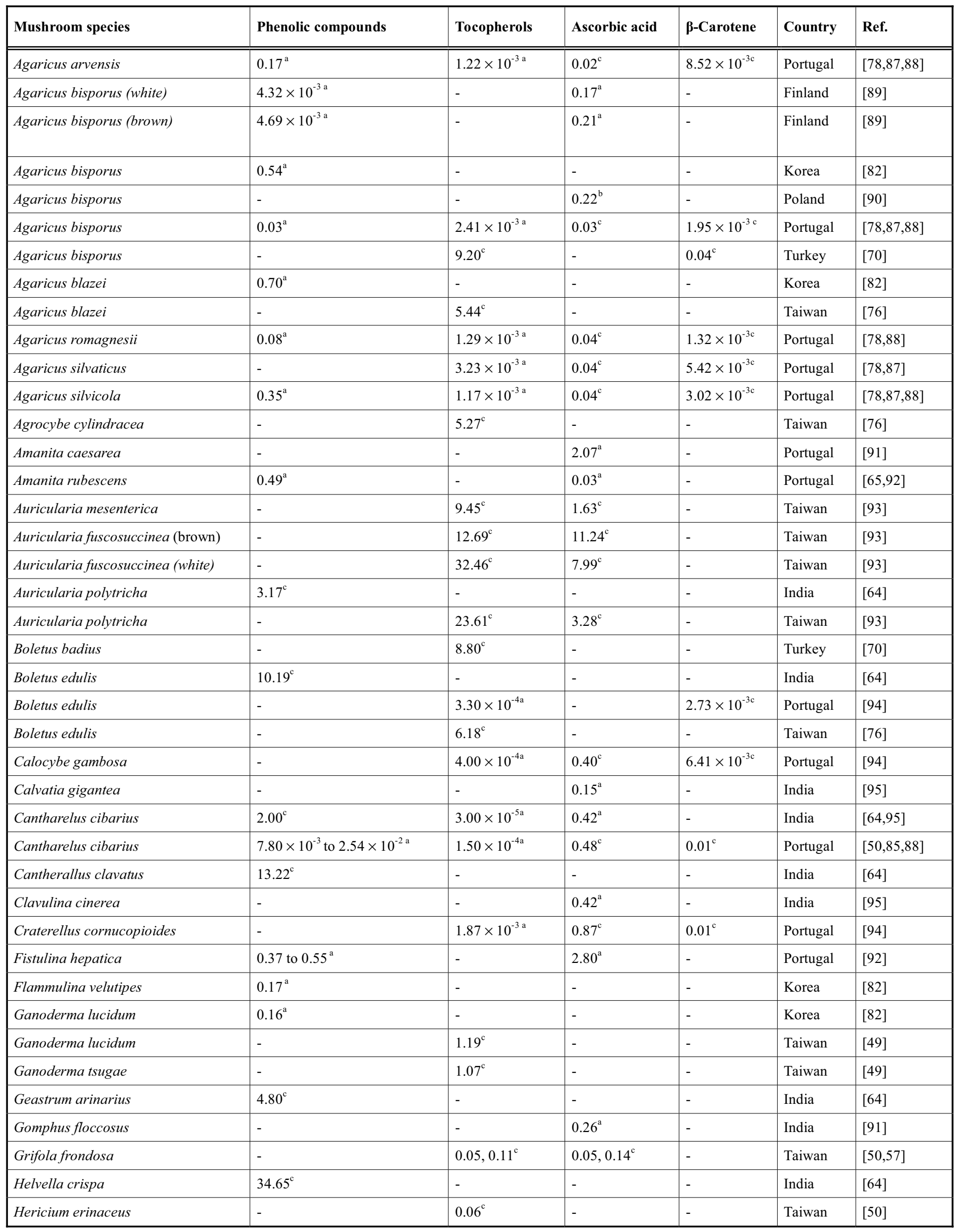


(Table 2). Contd....

\begin{tabular}{|c|c|c|c|c|c|c|}
\hline Mushroom species & Phenolic compounds & Tocopherols & Ascorbic acid & $\beta$-Carotene & Country & Ref. \\
\hline Hydnum repandum & $7.40^{\mathrm{c}}$ & - & - & - & India & {$[64]$} \\
\hline Hypholoma fasciculare & - & $6.00 \times 10^{-5 b}$ & $0.09^{\mathrm{c}}$ & $0.02^{\mathrm{c}}$ & Portugal & {$[79]$} \\
\hline Hypsizigus marmoreus & - & $2.96^{\mathrm{c}}$ & $0.13^{\mathrm{c}}$ & $0.02^{\mathrm{c}}$ & Taiwan & [74] \\
\hline Ionotus obliquus & $0.55^{\mathrm{a}}$ & - & - & - & Korea & {$[82]$} \\
\hline Lactarius deliciosus & $7.32^{\mathrm{c}}$ & - & - & - & India & {$[64]$} \\
\hline Lactarius deliciosus & $0.02^{\mathrm{a}}$ & - & 0.19 to $0.97^{\mathrm{a}}$ & $0.09^{c}$ & Portugal & {$[86,88,91]$} \\
\hline Lactarius piperatus & - & - & $0.16^{\mathrm{c}}$ & $0.03^{\mathrm{c}}$ & Portugal & {$[68]$} \\
\hline Lactarius quieticolor & - & - & $0.18^{\mathrm{a}}$ & - & India & {$[95]$} \\
\hline Lactarius sangifluus & $14.90^{\mathrm{c}}$ & - & - & - & India & {$[64]$} \\
\hline Lentinula edodes & - & $0.13^{\mathrm{c}}$ & - & - & Taiwan & {$[52]$} \\
\hline Lentinus edodes & $0.01^{\mathrm{a}}$ & - & $0.25^{\mathrm{a}}$ & - & Finland & [89] \\
\hline Lentinus edodes & $0.03^{\mathrm{a}}$ & - & - & - & Korea & {$[82]$} \\
\hline Lentinus sajor caju & $6.44^{\mathrm{c}}$ & - & - & - & India & {$[64]$} \\
\hline Lentinus squarrulosus & $15.00^{\mathrm{c}}$ & - & - & - & India & {$[64]$} \\
\hline Lepista nuda & $0.07^{\mathrm{a}}$ & $9.00 \times 10^{-5 b}$ & $0.23^{\mathrm{c}}$ & $2.52 \times 10^{-3 c}$ & Portugal & {$[79,88]$} \\
\hline Lepista nuda & - & $1.40^{\mathrm{c}}$ & - & $7.00 \times 10^{-3 c}$ & Turkey & {$[70]$} \\
\hline Leucopaxillus giganteus & - & - & $0.13^{\mathrm{c}}$ & $1.88 \times 10^{-3 c}$ & Portugal & {$[67]$} \\
\hline Lycoperdon molle & $0.08^{\mathrm{a}}$ & $3.00 \times 10^{-5 b}$ & $0.34^{\mathrm{c}}$ & $4.48 \times 10^{-3 c}$ & Portugal & {$[79,88]$} \\
\hline Lycoperdon perlatum & $0.01^{\mathrm{a}}$ & $3.00 \times 10^{-5 b}$ & $0.21^{\mathrm{c}}$ & $0.01^{\mathrm{c}}$ & Portugal & {$[79,88]$} \\
\hline Macrolepiota procera & $10.00^{\mathrm{c}}$ & - & - & - & India & {$[64]$} \\
\hline Macrolepiota procera & $0.02^{\mathrm{a}}$ & - & - & - & Portugal & [88] \\
\hline Marasmius oreades & - & $2.50 \times 10^{-4 a}$ & - & $1.99 \times 10^{-3 c}$ & Portugal & [94] \\
\hline Morchella anguiticeps & $12.92^{\mathrm{c}}$ & - & - & - & India & {$[64]$} \\
\hline Morchella conica & $16.90^{\mathrm{c}}$ & - & - & - & India & {$[64]$} \\
\hline Morchella esculenta & - & $0.07^{\mathrm{c}}$ & $0.13^{\mathrm{c}}$ & - & Taiwan & {$[57]$} \\
\hline Phellinus linteus & $0.21^{\mathrm{a}}$ & - & - & - & Korea & {$[82]$} \\
\hline Pleurotus eryngii & $0.03^{\mathrm{a}}$ & - & - & - & Korea & {$[82]$} \\
\hline Pleurotus cystidiosus & - & $0.45^{\mathrm{c}}$ & - & - & Taiwan & {$[52]$} \\
\hline Pleurotus djamor & $13.22^{\mathrm{c}}$ & - & - & - & India & {$[64]$} \\
\hline Pleurotus ostreatus & - & - & $0.20^{\mathrm{a}}$ & - & Finland & [89] \\
\hline Pleurotus ostreatus & $0.71^{\mathrm{a}}$ & $0.30^{\mathrm{c}}$ & $0.25^{\mathrm{c}}$ & $0.03^{\mathrm{c}}$ & India & {$[81]$} \\
\hline Pleurotus ostreatus & $0.09^{\mathrm{a}}$ & - & - & - & Korea & {$[82]$} \\
\hline Pleurotus ostreatus & - & $0.24^{\mathrm{c}}$ & - & - & Taiwan & {$[52]$} \\
\hline Pleurotus ostreatus & - & $0.90^{\mathrm{c}}$ & - & - & Turkey & {$[70]$} \\
\hline Pleurotus sajor-caju & $14.43^{\mathrm{c}}$ & - & - & - & India & {$[64]$} \\
\hline Polyporus squamosus & - & $0.30^{\mathrm{c}}$ & - & $0.02^{\mathrm{c}}$ & Turkey & {$[70]$} \\
\hline Ramaria botrytis & $0.36^{\mathrm{a}}$ & $2.50 \times 10^{-4 b}$ & $0.27^{\mathrm{c}}$ & $0.01^{\mathrm{c}}$ & Portugal & {$[79,88]$} \\
\hline Ramaria brevispora & - & - & $0.28^{\mathrm{a}}$ & - & India & {$[95]$} \\
\hline Russula brevepis & $5.50^{\mathrm{c}}$ & - & - & - & India & {$[64]$} \\
\hline Russula delica & - & $4.20^{\mathrm{c}}$ & - & $9.00 \times 10^{-3 c}$ & Turkey & {$[70]$} \\
\hline Russula integra & - & - & $0.20^{\mathrm{a}}$ & - & India & {$[95]$} \\
\hline Sarcodon imbricatus & $0.03^{\mathrm{a}}$ & - & $0.16^{\mathrm{c}}$ & $2.53 \times 10^{-3 c}$ & Portugal & {$[67,88]$} \\
\hline
\end{tabular}


(Table 2). Contd.....

\begin{tabular}{|c|c|c|c|c|c|c|}
\hline Sparassis crispa & $5.50^{\mathrm{c}}$ & - & - & - & India & {$[64]$} \\
\hline Suillus granulatus & $2.00 \times 10^{-3}$ to $1.59 \times 10^{-2 \mathrm{a}}$ & - & - & - & Portugal & {$[65,92]$} \\
\hline Suillus collinitus & - & - & 0.92 to $3.79^{\mathrm{a}}$ & - & Portugal & {$[91]$} \\
\hline Termitomyces albuminosus & - & $0.10^{\mathrm{c}}$ & $0.13^{\mathrm{c}}$ & - & Taiwan & {$[57]$} \\
\hline Termitomyces heimii & $37.00^{\mathrm{c}}$ & - & - & - & India & {$[64]$} \\
\hline Termitomyces microcarpus & $6.73^{\mathrm{c}}$ & - & - & - & India & {$[64]$} \\
\hline Termitomyces mummiformis & $19.20^{\mathrm{c}}$ & - & - & - & India & {$[64]$} \\
\hline Tricholoma acerbum & $0.04^{\mathrm{a}}$ & $8.00 \times 10^{-5 b}$ & $0.22^{\mathrm{c}}$ & $0.08^{\mathrm{c}}$ & Portugal & {$[79,88]$} \\
\hline Tricholoma equestre & $0.04,0.12^{\mathrm{a}}$ & - & - & - & Portugal & {$[65]$} \\
\hline Trichloma giganteum & - & $0.12^{\mathrm{c}}$ & - & - & Taiwan & {$[50]$} \\
\hline Verpa conica & - & $1.90^{\mathrm{c}}$ & - & $0.01^{\mathrm{c}}$ & Turkey & {$[70]$} \\
\hline
\end{tabular}

Values are Presented in: ${ }^{a} \mathrm{mg} / \mathrm{g}$ of Dry Weight; ${ }^{\mathrm{b}} \mathrm{mg} / \mathrm{g}$ of Fresh Weight; ${ }^{\mathrm{c}} \mathrm{mg} / \mathrm{g}$ of Extract.

Table 3. Phenolic Compounds Detected in Wild Mushrooms

\begin{tabular}{|c|c|c|c|}
\hline Phenolic compound & Mushroom species & Country & Ref. \\
\hline Benzoic acid & Agaricus blazei, Sparassis crispa, Phellinus linteus & Korea & {$[82]$} \\
\hline \multirow{5}{*}{$\begin{array}{l}p \text {-Hydroxybenzoic } \\
\text { acid }\end{array}$} & Agaricus bisporus (white), Agaricus bisporus (brown), Lentinus edodes & Finland & {$[89]$} \\
\hline & Amanita rubescens, Russula cyanoxantha, Tricholoma equestre & Portugal & {$[65]$} \\
\hline & Amanita rubescens, Suillus granulatus & Portugal & {$[92]$} \\
\hline & $\begin{array}{l}\text { Agaricus arvensis, Agaricus bisporus, Agaricus silvicola, Agaricus romagnesii, Lactarius deliciosus, } \\
\text { Lepista nuda, Lycoperdon molle, Sarcodon imbricatus, Ramaria botrytis, Tricholoma acerbum }\end{array}$ & Portugal & {$[88]$} \\
\hline & Sparassis crispa, Phellinus linteus, Ionotus obliquus & Korea & {$[82]$} \\
\hline \multirow[t]{4}{*}{ Protocatechuic acid } & Agaricus bisporus (white), Agaricus bisporus (brown), Lentinus edodes & Finland & [89] \\
\hline & $\begin{array}{l}\text { Termitomyces heimii, Termitomyces mummiformis, Boletus edulis, Lactarius deliciosus, Pleurotus } \\
\text { sajor-caju, Hydnum repandum, Lentinus squarrulosus, Sparassis crispa, Morchella conica, Russula } \\
\text { brevepis, Lactarius sangifluus, Macrolepiota procera, Cantherallus clavatus, Auricularia polytricha, } \\
\text { Pleurotus djamor, Lentinus sajor caju, Termitomyces tylerance, Morchella anguiticeps, Termitomyces } \\
\text { microcarpus, Helvella crispa, Termitomyces shimperi }\end{array}$ & India & {$[64]$} \\
\hline & Lepista nuda, Ramaria botrytis & Portugal & {$[88]$} \\
\hline & $\begin{array}{l}\text { Pleurotus ostreatus, Agaricus bisporus, Flammulina velutipes, Pleurotus eryngii, Lentinus edodes, } \\
\text { Agaricus blazei, Sparassis crispa, Phellinus linteus, Ganoderma lucidum, Ionotus obliquus }\end{array}$ & Korea & {$[82]$} \\
\hline \multirow[t]{2}{*}{ Gallic acid } & $\begin{array}{l}\text { Termitomyces heimii, Termitomyces mummiformis, Lactarius deliciosus, Pleurotus sajor-caju, Hydnum } \\
\text { repandum, Lentinus squarrulosus, Sparassis crispa, Morchella conica, Russula brevepis, Geastrum } \\
\text { arinarius, Cantharellus cibarius, Lactarius sangifluus, Macrolepiota procera, Cantherallus clavatus, } \\
\text { Auricularia polytricha, Pleurotus djamor, Lentinus sajor caju, Termitomyces tylerance, Morchella } \\
\text { anguiticeps, Termitomyces Microcarpus, Helvella crispa, Termitomyces shimperi }\end{array}$ & India & {$[64]$} \\
\hline & $\begin{array}{l}\text { Pleurotus ostreatus, Agaricus bisporus, Flammulina velutipes, Pleurotus eryngii, Lentinus edodes, } \\
\text { Agaricus blazei, Sparassis crispa, Phellinus linteus, Ganoderma lucidum, Ionotus obliquus }\end{array}$ & Korea & {$[82]$} \\
\hline \multirow[t]{2}{*}{ Gentisic acid } & $\begin{array}{l}\text { Termitomyces heimii, Termitomyces mummiformis, Lactarius deliciosus, Pleurotus sajor-caju, Hydnum } \\
\text { repandum, Lentinus squarrulosus, Sparassis crispa, Morchella conica, Russula brevepis, Lactarius } \\
\text { sangifluus, Macrolepiota procera, Cantherallus clavatus, Auricularia polytricha, Pleurotus djamor, } \\
\text { Termitomyces tylerance, Morchella anguiticeps, Termitomyces Microcarpus, Helvella crispa, } \\
\text { Termitomyces shimperi }\end{array}$ & India & {$[64]$} \\
\hline & Agaricus blazei & Korea & [82] \\
\hline
\end{tabular}


(Table 3). Contd.....

\begin{tabular}{|c|c|c|c|}
\hline Phenolic compound & Mushroom species & Country & Ref. \\
\hline Homogentisic acid & Pleurotus ostreatus, Flammulina velutipes, Ionotus obliquus & Korea & {$[82]$} \\
\hline \multirow[t]{2}{*}{ Vanillic acid } & $\begin{array}{l}\text { Termitomyces heimii, Pleurotus sajorcaju, Hydnum repandum, Lentinus squarrulosus, Morchella } \\
\text { conica, Russula brevepis, Lactarius sangifluus, Macrolepiota procera, Cantherallus clavatus, } \\
\text { Auricularia polytricha, Pleurotus djamor, Lentinus sajor caju, Termitomyces Microcarpus, Helvella } \\
\text { crispa, Termitomyces shimperi }\end{array}$ & India & {$[64]$} \\
\hline & Lycoperdon molle, Tricholoma acerbum & Portugal & {$[88]$} \\
\hline 5-Sulfosalicylic acid & Flammulina velutipes, Sparassis crispa, Phellinus linteus, Ganoderma lucidum & Korea & {$[82]$} \\
\hline \multirow[t]{2}{*}{ Syringic acid } & $\begin{array}{l}\text { Termitomyces mummiformis, Hydnum repandum, Morchella conica, Russula brevepis, Lactarius } \\
\text { sangifluus, Macrolepiota procera, Cantherallus clavatus, Pleurotus djamor, Lentinus sajor caju, } \\
\text { Termitomyces tylerance, Morchella anguiticeps, Termitomyces Microcarpus }\end{array}$ & India & {$[64]$} \\
\hline & Agaricus blazei, Sparassis crispa & Korea & {$[82]$} \\
\hline Veratric acid & Sparassis crispa & Korea & {$[82]$} \\
\hline Vanillin & Ionotus obliquus & Korea & {$[82]$} \\
\hline \multirow[t]{4}{*}{ Cinnamic acid } & Agaricus bisporus (white), Agaricus bisporus (brown), Lentinus edodes & Finland & {$[89]$} \\
\hline & $\begin{array}{l}\text { Termitomyces heimii, Termitomyces mummiformis, Pleurotus sajor-caju, Hydnum repandum, Lentinus } \\
\text { squarrulosus, Sparassis crispa, Lactarius sangifluus, Cantherallus clavatus, Pleurotus djamor, } \\
\text { Termitomyces shimperi }\end{array}$ & India & {$[64]$} \\
\hline & $\begin{array}{l}\text { Agaricus arvensis, Agaricus bisporus, Agaricus silvicola, Agaricus romagnesii, Cantharelus cibarius, } \\
\text { Lycoperdon perlatum, Macrolepiota procera }\end{array}$ & Portugal & {$[88]$} \\
\hline & Agaricus blazei & Korea & {$[82]$} \\
\hline \multirow[t]{5}{*}{$p$-Coumaric acid } & Cantharellus cibarius & Portugal & {$[85]$} \\
\hline & $\begin{array}{l}\text { Termitomyces heimii, Boletus edulis, Sparassis crispa, Geastrum arinarius, Cantharellus cibarius, } \\
\text { Lactarius sangifluus, Macrolepiota procera, Pleurotus djamor, Lentinus sajor caju }\end{array}$ & India & {$[64]$} \\
\hline & Fistulina hepatica & Portugal & {$[92]$} \\
\hline & Agaricus arvensis, Agaricus silvicola, Lepista nuda & Portugal & {$[88]$} \\
\hline & Sparassis crispa & Korea & {$[82]$} \\
\hline$o$-Coumaric acid & Ionotus obliquus & Korea & {$[82]$} \\
\hline \multirow[t]{5}{*}{ Caffeic acid } & Sparassis crispa & Korea & {$[82]$} \\
\hline & Cantharellus cibarius & Portugal & {$[85]$} \\
\hline & $\begin{array}{l}\text { Termitomyces heimii, Boletus edulis, Lentinus squarrulosus, Morchella conica, Russula brevepis, } \\
\text { Cantharellus cibarius, Lactarius sangifluus, Macrolepiota procera, Cantherallus clavatus, Pleurotus } \\
\text { djamor, Lentinus sajor caju, Termitomyces tylerance, Morchella anguiticeps, Termitomyces } \\
\text { Microcarpus, Termitomyces shimperi }\end{array}$ & India & {$[64]$} \\
\hline & Fistulina hepatica & Portugal & {$[92]$} \\
\hline & Flammulina velutipes, Sparassis crispa, Phellinus linteus & Korea & {$[82]$} \\
\hline \multirow[t]{2}{*}{ Ferulic acid } & $\begin{array}{l}\text { Termitomyces heimii, Lactarius deliciosus, Pleurotus sajor-caju, Lentinus squarrulosus, Sparassis } \\
\text { crispa, Morchella conica, Cantharellus cibarius, Lactarius sangifluus, Macrolepiota procera, } \\
\text { Cantherallus clavatus, Pleurotus djamor, Termitomyces Microcarpus, Termitomyces shimperi }\end{array}$ & India & {$[64]$} \\
\hline & Flammulina velutipes, Ionotus obliquus & Korea & {$[82]$} \\
\hline 3-O-Caffeoylquinic acid & Cantharellus cibarius & Portugal & {$[85]$} \\
\hline 4-O-Caffeoylquinic acid & Cantharellus cibarius & Portugal & {$[85]$} \\
\hline \multirow[t]{2}{*}{ 5-O-Caffeoylquinic acid } & Cantharellus cibarius & Portugal & {$[85]$} \\
\hline & Pleurotus ostreatus, Flammulina velutipes, Phellinus linteus & Korea & {$[82]$} \\
\hline \multirow[t]{2}{*}{ Quercetin } & Suillus luteus, Suillus granulatus & Portugal & {$[65]$} \\
\hline & Flammulina velutipes, Agaricus blazei, Sparassis crispa, Ganoderma lucidum, Ionotus obliquus & Korea & {$[82]$} \\
\hline \multirow[t]{2}{*}{ Rutin } & Cantharellus cibarius & Portugal & {$[85]$} \\
\hline & Pleurotus ostreatus & India & {$[81]$} \\
\hline Kaempferol & Sparassis crispa, Ganoderma lucidum, Ionotus obliquus & Korea & {$[82]$} \\
\hline
\end{tabular}


(Table 3). Contd.....

\begin{tabular}{|c|c|c|c|}
\hline Phenolic compound & Mushroom species & Country & Ref. \\
\hline Myricetin & Pleurotus ostreatus, Agaricus bisporus, Agaricus blazei, Ganoderma lucidum & Korea & {$[82]$} \\
\hline Chrysin & Pleurotus ostreatus & India & [81] \\
\hline Hesperetin & Ganoderma lucidum & Korea & {$[82]$} \\
\hline Naringenin & Sparassis crispa & Korea & [82] \\
\hline Formometin & Ganoderma lucidum & Korea & [82] \\
\hline Biochanin & Ganoderma lucidum & Korea & [82] \\
\hline Pyrogallol & $\begin{array}{l}\text { Agaricus bisporus, Flammulina velutipes, Agaricus blazei, Sparassis crispa, Ganoderma lucidum, } \\
\text { Phellinus linteus }\end{array}$ & Korea & [82] \\
\hline
\end{tabular}

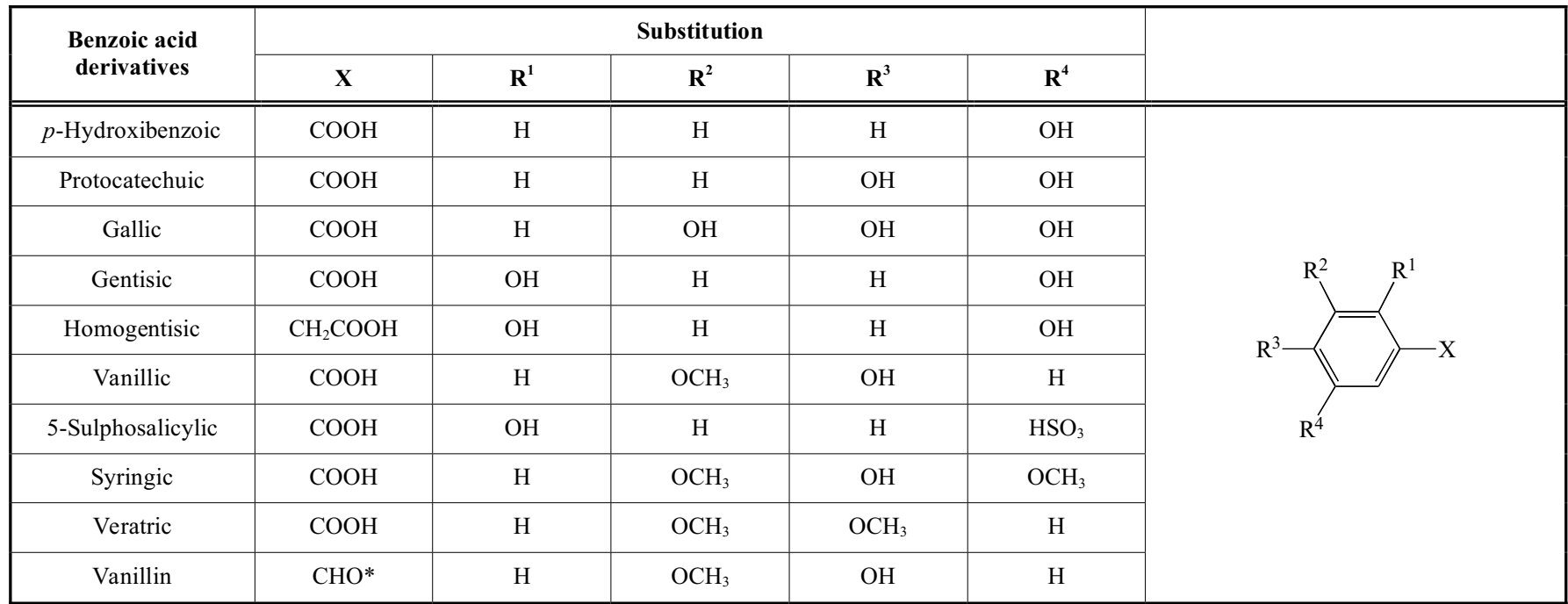

Fig. (3). Chemical structure of the benzoic acid derivatives found in mushrooms.

* Aldeydes are groups under the corresponding phenolic acid class.

Hydroxybenzoic acid derivatives commonly occur in the bound form and are typically a component of a complex structure like lignins and hydrolyzable tannins. They can also be found linked to sugars or organic acids in plant foods. Hydroxycinnamic acid derivatives are mainly present in the bound form, linked to cell-wall structural components, such as cellulose, lignin, and proteins, as well as associated to organic acids, such as tartaric or quinic acids (i.e., chlorogenic acids), through ester bonds [38].

The overall effectiveness of a natural phenolic antioxidant depends on the involvement of the phenolic hydrogen in radical reactions, the stability of the natural antioxidant radical formed during radical reactions, and the chemical substitutions present on the structure [99]. The substitutions on the structure are probably the most significant with respect to the ability of a natural antioxidant to participate in the control of radical reactions and to form resonance-stabilized natural antioxidant radicals. The role of phenolic antioxidants $(\mathrm{ArOH})$ is to interrupt the chain reaction according to:

$$
\mathrm{RO}_{2}^{\circ}+\mathrm{ArOH} \longrightarrow \mathrm{ROOH}+\mathrm{ArO}^{\bullet}
$$

To be effective $\mathrm{ArO}^{\circ}$ must be a relatively stable free radical, so that it reacts slowly with substrate $\mathrm{RH}$ but rapidly with $\mathrm{RO}_{2}{ }^{\circ}$, hence the term "chain-breaking antioxidant" [100].

Like other phenolic compounds, the antioxidant activity of phenolic acids is due to the phenolic hydrogens. Hydroxyl substitutions at ortho and para positions also will enhance antioxidant activity. Intramolecular hydrogen bonds are formed by ortho substituted phenols (e.g., 1, 2-dihydroxybenzene) during radical reactions, which increase the stabil- 


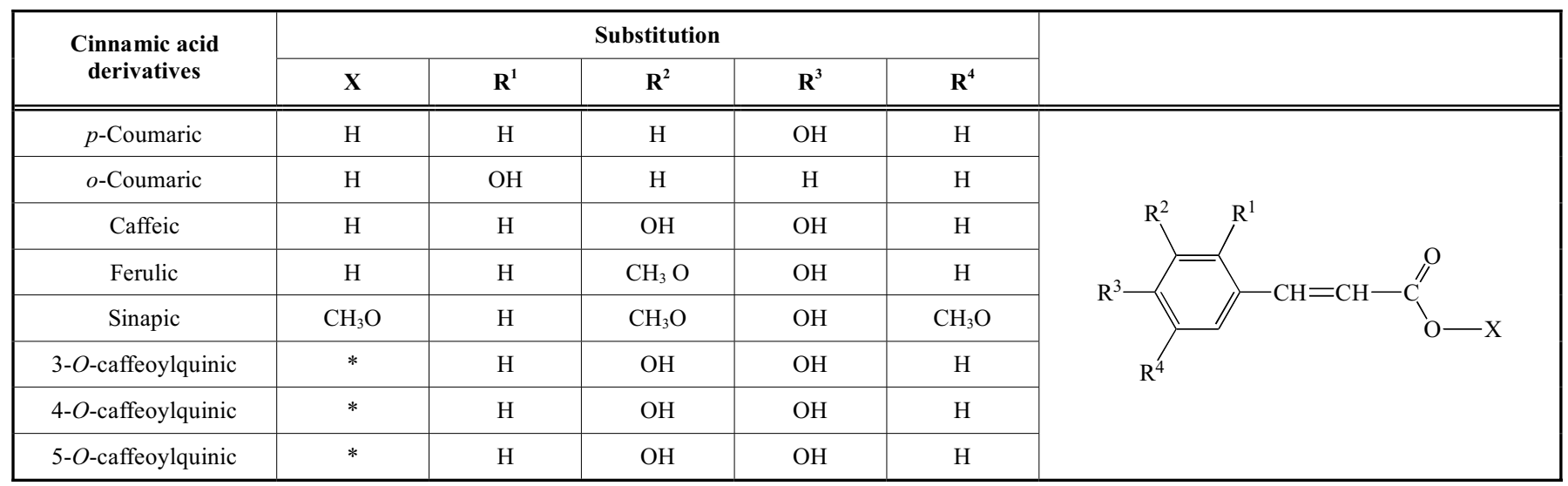

Fig. (4). Chemical structure of the cinnamic acid derivatives found in mushrooms.

* The carboxylic group is esterified with quinic acid.

ity of the phenoxy radical. It has been reported that caffeic acid is a better antioxidant than ferulic acid or $p$-coumaric acid. The second hydroxy group at ortho position allows the formation of intramolecular hydrogen bonds, which results in stronger antioxidant activity than those of compounds containing a methoxy $\left(\mathrm{OCH}_{3}\right)$ substitution ortho to the hydroxy group. Ferulic acid contains ortho methoxy substitution in its structure that may provide a stabilizing effect on the phenoxyl radical, which enhances its antioxidant activity over $p$-coumaric acid. The higher antioxidant activity of trihydroxybenzoic acid (i.e., gallic acid) over 3,4- dihydroxybenzoic acid (i.e., protocatechuic acid) is due to the presence of three hydroxyl groups in trihydroxybenzoic acid [99, $101]$.

The acid proton appears to have little impact on antioxidant activity. Both caffeic acid and chlorogenic acid, the resultant compound after replacement of the acid proton of caffeic acid with quinic acid via an ester bond, were equally effective in controlling lipid oxidation [99]. The allylic group, as found in cinnamic acid derivatives, provides enhanced antioxidant activity when compared to benzoic acid derivatives. Caffeic acid (3,4- dihydroxycinnamic acid) was reported to be a better antioxidant than protocatechuic acid (3, 4-dihydroxybenzoic acid) in a lard system. The allylic group may improve the resonance stability of the phenoxyl radical [102].

In general, it is assumed that only plants possess the biosynthetic ability to produce flavonoids and not animals and fungi. Even though some flavonoids have exceptionally been reported from fungi Aspergillus candidus and Phallus impudicus [103] and more recently in mushrooms (Table 3). Recently, Barros et al. [88] reported that no flavonoids were detected in sixteen Portuguese wild mushrooms.

Flavonoids represent a large group of phenolic compounds with antioxidant activity, that occur naturally in plants and are found in fruits, vegetables, grains, barks, roots, stems, flowers, and derived products like tea and wine. These compounds have been linked to reduce the risk of major chronic diseases [38]. They are characterized for the carbon skeleton $\mathrm{C} 6-\mathrm{C} 3-\mathrm{C} 6$. The basic structure of these compounds consists of two aromatic rings ( $\mathrm{A}$ and $\mathrm{B}$ rings) linked by a three carbon chain that is usually in an oxygenated heterocycle ring, or C ring (Fig. 5) [103].

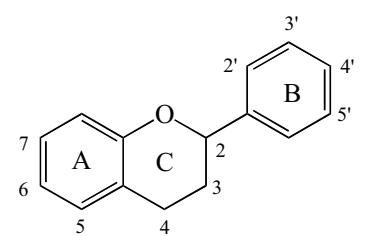

Fig. (5). The generic structure of flavonoids.

Several classes of flavonoids are delineated on the basis of differences in the generic structure of the heterocycle C ring and can be classified into flavonols, flavones, flavanols, flavanones, anthocyanins and isoflavonoids [104]. Fig. (6) presents the chemical structure of the flavonoids identified in wild mushrooms.

Flavonoids are most frequently found in nature as conjugates in glycosylated or esterified forms but can also occur in food as aglycones, especially as a result of the effects of food processing. Flavonols are the most abundant flavonoids in foods [105].

Multiple mechanisms have been identified as involved in the health-promoting effects of flavonoids, including antioxidant, anti-inflammatory and anti-proliferative activities, inhibition of bioactivating enzymes, or induction of detoxifying enzymes [106]. The antioxidant property of flavonoids was the first mechanism of action studied, in particular with regard to their protective effect against cardiovascular diseases. Flavonoids have been shown to be highly effective scavengers of most types of oxidizing molecules, including singlet oxygen and various free radicals, which are possibly involved in DNA damage and tumor promotion [100].

Phenolic compounds have specific health effects, even though they are non-nutritive compounds. In our diet they might provide health benefits associated with reduced risk of chronic diseases that may be due to their ability to reduce agents by donating hydrogen and quenching singlet oxygen. Antioxidant properties of phenolic compounds also play a vital role in the stability of food products, as well as in the antioxidative defence mechanisms of biological systems [107].

\section{Tocopherols}

Vitamin $\mathrm{E}$ is a term frequently used to designate a family of chemically related compounds, namely tocopherols and 
<smiles>[Z17]c1cc(-c2oc3cc(O)cc(O)c3c(=O)c2O)cc([Z13])c1[R1]</smiles>

Flavonols<smiles>[Z13]c1cc(-c2cc(=O)c3c(O)cc(O)cc3o2)cc([R1])c1[R1]</smiles>

Flavones<smiles>[Z13]c1cc(C2Oc3cc(O)cc(O)c3CC2O)cc([Z13])c1[R1]</smiles>

Flavan-3-oles<smiles>[Z17]c1cc(C2CC(=O)c3c(O)cc(O)cc3O2)cc([R1])c1[R1]</smiles>

Flavanones<smiles>[R1]c1cc(-c2coc3cc(O)cc([R1])c3c2=O)cc([Y13])c1[Z17]</smiles>

Isoflavones

Fig. (6). Chemical structures of several flavonoids found in wild mushrooms. Flavonols: Quercetin $\left(\mathrm{R}_{1}^{\prime}=\mathrm{R}_{2}^{\prime}=\mathrm{OH}, \mathrm{R}_{3}=\mathrm{H}^{\prime}\right), \mathrm{Rutin}^{\prime}$ $\left(\mathrm{R}_{1}^{\prime}=\mathrm{R}_{2}^{\prime}=\mathrm{OH}, \mathrm{R}_{3}=\mathrm{H}\right.$; OH in position-3 is substituted with the disaccharide rutinose), Kaempferol $\left(\mathrm{R}_{1}^{\prime}=\mathrm{R}_{3}^{\prime}=\mathrm{H}, \mathrm{R}_{2}^{\prime}=\mathrm{OH}\right), \mathrm{Myricetin}$ $\left(\mathrm{R}_{1}^{\prime}=\mathrm{R}_{2}{ }_{2}=\mathrm{R}_{3}{ }_{3}=\mathrm{OH}\right)$. Flavones: Chrysin $\left(\mathrm{R}_{1}{ }_{1}=\mathrm{R}_{2}{ }_{2}=\mathrm{R}_{3}{ }_{3}=\mathrm{H}\right)$. Flavan-3-oles: Catechin $\left(\mathrm{R}_{1}{ }_{1}=\mathrm{H}, \mathrm{R}_{2}{ }_{2}=\mathrm{R}_{3}{ }_{3}=\mathrm{OH}\right)$. Flavanones: Hesperetin $\left(\mathrm{R}_{1}{ }_{1}=\mathrm{OH}\right.$, $\left.\mathrm{R}_{2}{ }_{2}=\mathrm{R}_{3}^{\prime}=\mathrm{H}\right)$, Naringenin $\left(\mathrm{R}_{1}{ }_{1}=\mathrm{R}_{3}{ }_{3}=\mathrm{H}_{,}, \mathrm{R}_{2}=\mathrm{OH}\right)$, Naringin $\left(\mathrm{R}_{1}^{\prime}=\mathrm{R}_{3}^{\prime}=\mathrm{H}, \mathrm{R}_{2}=\mathrm{OH}\right.$; OH in position-7 is substituted with the disaccharide rutinose). Isoflavones: Formonetim $\left(\mathrm{R}^{1}=\mathrm{H}, \mathrm{R}_{1}{ }_{1}=\mathrm{R}_{3}{ }_{3}=\mathrm{H}, \mathrm{R}_{2}{ }_{2}=\mathrm{OCH}_{3}\right)$, Biochanin $\left(\mathrm{R}^{1}=\mathrm{OH}, \mathrm{R}_{1}=\mathrm{R}_{3}{ }_{3}=\mathrm{H}, \mathrm{R}_{2}{ }_{2}=\mathrm{OCH}_{3}\right)$.

tocotrienols, which share a common structure with a chromanol head and isoprenic side chain. Vitamin E is composed of eight chemical compounds: $\alpha-, \beta-, \gamma-$ and $\delta$ - tocopherols and four corresponding tocotrienols (Fig. 7) [108]. It is an important natural antioxidant in foods, especially those rich in polyunsaturated fatty acids. Due to its role as a scavenger of free radicals, vitamin $\mathrm{E}$ is also believed to protect our bodies against degenerative malfunctions, mainly cancer and cardiovascular diseases [109, 110].

Some reports have been published on the tocopherols content of mushrooms (Table 4). All reported the same methodology including saponification in the extraction process and analysis by HPLC coupled to UV detector. Only Barros et al. [87] described an extraction process without saponification, adding an antioxidant to avoid tocopherols oxidation, using special precautions to protect the samples from light and heat, and a fluorescence detector. $\alpha-, \beta-, \delta$ - and $\gamma$-Tocopherols were identified and quantified in wild mushrooms, while tocotrienols were not detected in any of the cited studies.

Vitamin E reacts with peroxyl radicals produced from polyunsaturated fatty acids in membrane phospholipids or lipoproteins to yield a stable lipid hydroperoxide. They act as antioxidants by donating a hydrogen atom to peroxyl radicals of unsaturated lipid molecules, forming a hydroperoxide and a tocopheroxyl radical, which reacts with other peroxyl or tocopheroxyl radicals forming more stable adducts [111].

$$
\begin{aligned}
& \mathrm{LH}+\text { Oxidant initiator } \longrightarrow \mathrm{L}^{\circ} \\
& \mathrm{L}^{\circ}+\mathrm{O}_{2} \longrightarrow \mathrm{LOO}^{\circ} \\
& \mathrm{LOO}^{\bullet}+\text { Tocopherol } \longrightarrow \mathrm{LOOH}+\text { Tocopherol }^{\bullet}
\end{aligned}
$$

In the past $\alpha$-tocopherol was considered the most active form of vitamin $\mathrm{E}$ in humans and it was reported to exhibit<smiles>[R]c1c(C)c2c(c([R])c1O)CCC(C)(CCCC(C)CCCC(C)CCCC(C)C)O2</smiles><smiles>[R]c1c(C)c2c(c([R])c1O)CCC(C)(CC/C=C(/C)CC/C=C(\C)CCC=C(C)C)O2</smiles>

$\mathrm{R}^{1}=\mathrm{R}^{2}=$ Me- $\alpha$-tocopherol

$\mathrm{R}^{1}=\mathrm{Me}, \mathrm{R}^{2}=\mathrm{H}-\beta$-tocopherol

$\mathrm{R}^{1}=\mathrm{H}, \mathrm{R}^{2}=$ Me- $\gamma$-tocopherol

$\mathrm{R}^{1}=\mathrm{R}^{2}=\mathrm{H}-\delta$-tocopherol

$$
\begin{aligned}
& \mathrm{R}^{1}=\mathrm{R}^{2}=\text { Me- } \alpha \text {-tocotrienol } \\
& \mathrm{R}^{1}=\mathrm{Me}, \mathrm{R}^{2}=\mathrm{H}-\beta \text {-tocotrienol } \\
& \mathrm{R}^{1}=\mathrm{H}, \mathrm{R}^{2}=\text { Me- } \gamma \text {-tocotrienol } \\
& \mathrm{R}^{1}=\mathrm{R}^{2}=\mathrm{H}-\delta \text {-tocotrienol }
\end{aligned}
$$

Fig. (7). Chemical structure of tocopherols and tocotrienols. 
Table 4. Tocopherols Detected in Wild Mushrooms

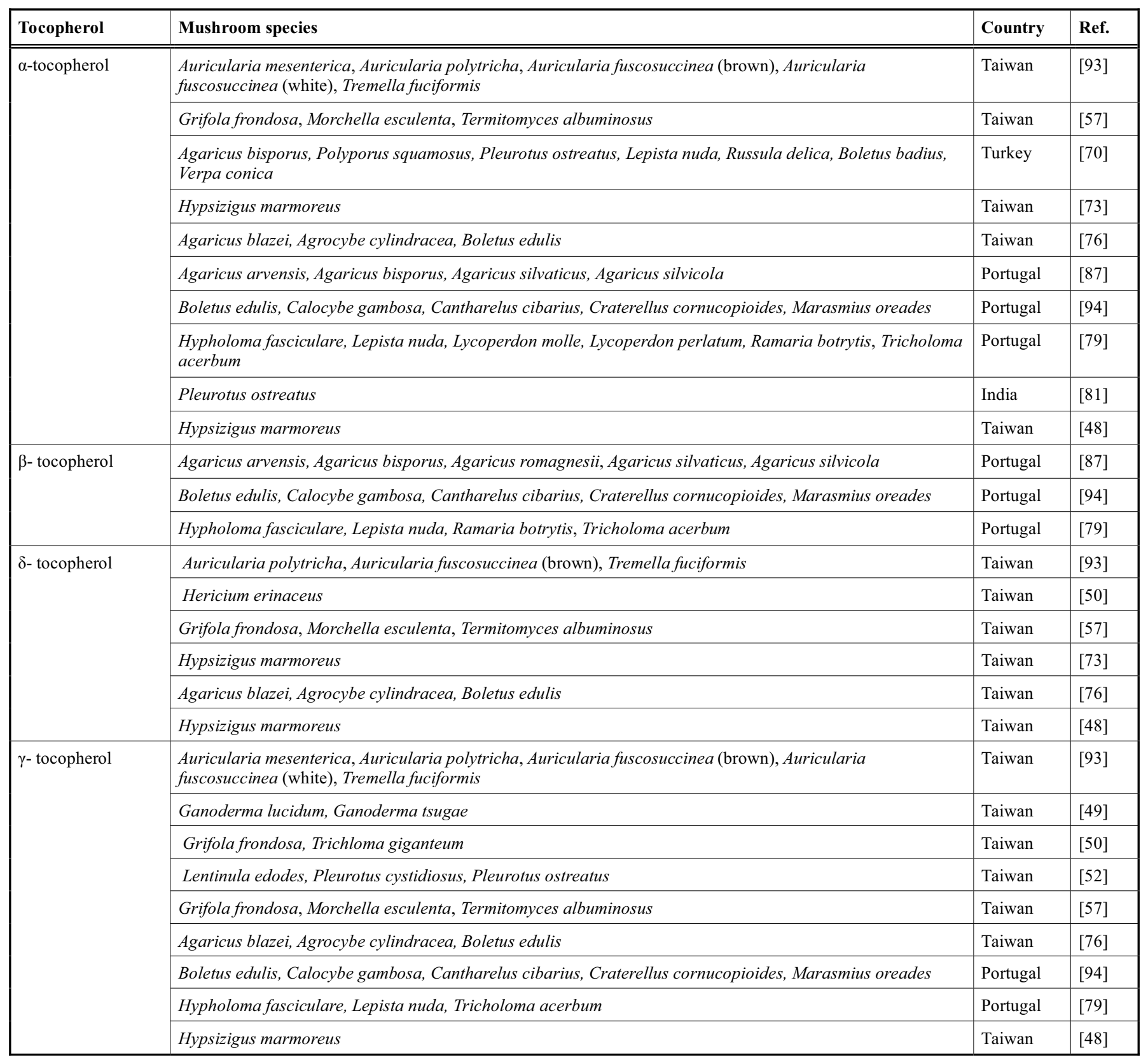

the highest biological activity. However, many recent publications have been focused on the health effects of the other vitamin $\mathrm{E}$ isoforms $[112,113]$.

\section{Ascorbic Acid}

Ascorbic acid, also known as vitamin $\mathrm{C}$, is related to the C6 sugars, being the aldono-1,4-lactone of a hexonic acid (Lgalactonic or L-gulonic acid), and contains an enediol group on carbons 2 and 3 (Fig. 8); exists in two stereoisomeric forms: a L-isomer and a D-isomer [114]. It is a necessary nutrient for a limited number of animals, including humans, that are incapable of its synthesis and that must secure vitamin $\mathrm{C}$ by means of dietary uptake $[115,116]$. In addition, ascorbic acid is thought to exert a protective role against various oxidative stress-related diseases such as heart dis- ease, stroke, cancer, several neurodegenerative diseases and cataractogenesis [12].<smiles>O=C1OC(C(O)CO)C(O)=C1O</smiles>

Fig. (8). Chemical structure of ascorbic acid.

Vitamin $\mathrm{C}$, one of the simplest vitamins, was found in several mushroom species (Table 5), either using HPLC coupled to UV or fluorescence detector, or following the spectrophotometer procedure based on the reaction with 2,6dichlorophenolindophenol [117]. 
Table 5. Studies Reporting the Presence of Ascorbic Acid in Wild Mushrooms

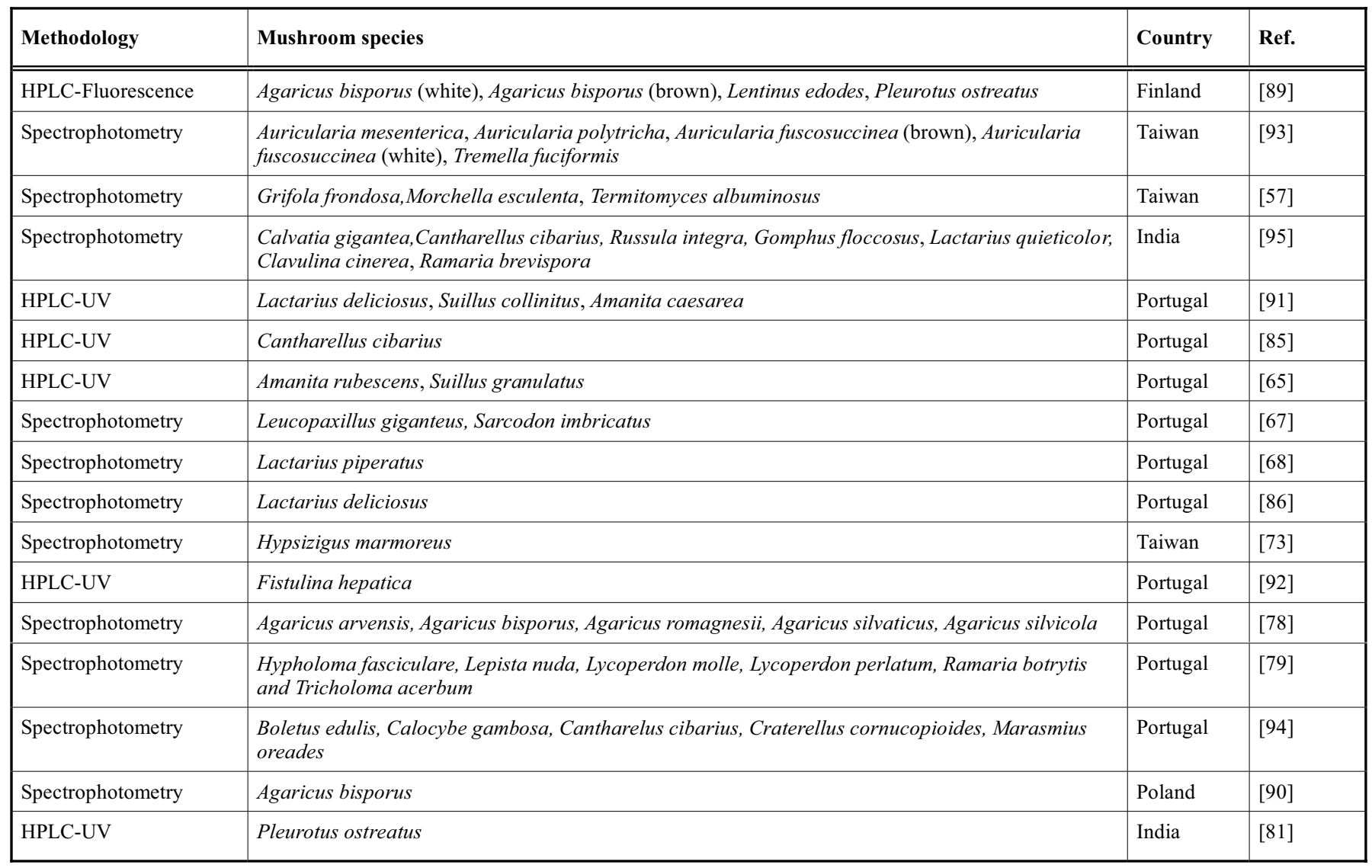

Vitamin $\mathrm{C}$ can protect biomembranes against lipid peroxidation damage by eliminating peroxyl radicals in the aqueous phase before the latter can initiate lipid peroxidation [114]. Vitamin $\mathrm{C}$ is effective against superoxide, hydroxyl radical, hydrogen peroxide, peroxyl radical and singlet oxygen [118]. Cooperative interactions exist among vitamin C and vitamin E. They interact synergistically at the membrane-cytosol interface to regenerate membrane-bound oxidized vitamin E [119] (Fig. 2). As a lipophilic antioxidant, vitamin $\mathrm{E}$ can interact with the lipid components in the vascular systems, notably LDL, and protects them from atherogenic oxidative modification [120]. Conversely, the lipidbound $\alpha$-tocopherols can be oxidized by aqueous-phase radicals and transformed into reactive tocopherol radicals, which, in turn, react with the unsaturated lipids of the lipoprotein, initiating lipid oxidation by a tocopherol-mediated peroxidation reaction. Oxidized vitamin E can be reduced back to its antioxidant form by other aqueous-phase reductants, like ascorbic acid [121].

Ascorbic acid reacts rapidly with the tocopherol radical by reducing the ascorbate radical (semidehydroascorbate) to ascorbate by NADH-dependent semidehydroascorbate reductase.

$$
\begin{aligned}
\text { Ascobic acid }+ \text { Vitamin } \mathrm{E}^{\bullet} & \longrightarrow \text { Ascorbate }+ \text { Vitamin } \mathrm{E} \\
\text { Ascobate }+\mathrm{NADH} & \longrightarrow \text { Ascorbate }+\mathrm{NAD}^{\bullet}
\end{aligned}
$$

In addition, ascorbate may sequester aqueous radicals in the plasma before they can oxidize vitamin $\mathrm{E}$ in the lipid phase and affords protection for lipid-bound tocopherols. The interactions among these antioxidant nutrients are likely very important in protecting cells because the concentration of each antioxidant alone may not be adequate to effectively protect these cells against lipid peroxidation $[122,123]$.

\section{Carotenoids}

Carotenoids are nature's most widespread pigments and have also received substantial attention because of both their provitamin and antioxidant roles [38]. Particularly, $\beta$ carotene was found in several mushroom species (Table 6) either using HPLC coupled to UV or fluorescence detector, or following spectrophotometer procedures.

Carotenoids are synthesized by plants and microorganisms but not animals. Fruits and vegetables constitute the major sources of carotenoids in human diet [124, 125]. Close to $90 \%$ of the carotenoids in the diet and human body are represented by $\beta$-carotene, $\alpha$-carotene, lycopene, lutein and $\beta$-cryptoxanthin [126] (Fig. 9).

All the carotenoids posses a 40-carbon skeleton of polyisoprenoid structure, a long conjugated double bonds chain forming the central part of the molecule and a near bilateral symmetry around the central double bond, as common chemical features [127]. This gives them their shape, chemical reactivity, and light-absorbing properties. Different carotenoids are derived essentially by modifications in the base structure by cyclization of the end groups and by introduction of oxygen-containing functional groups, giving them 
Table 6. Studies Reporting the Presence of $\beta$-Carotene in Wild Mushrooms

\begin{tabular}{|c|c|c|c|}
\hline Methodology & Mushroom species & Country & Ref. \\
\hline Spectrophotometry & Leucopaxillus giganteus, Sarcodon imbricatus & Portugal & {$[67]$} \\
\hline Spectrophotometry & Lactarius piperatus & Portugal & {$[68]$} \\
\hline Spectrophotometry & Lactarius deliciosus & Portugal & {$[86]$} \\
\hline HPLC-UV & Agaricus bisporus, Polyporus squamosus, Lepista nuda, Russula delica, Verpa conica & Turkey & {$[70]$} \\
\hline Spectrophotometry & Agaricus arvensis, Agaricus bisporus, Agaricus romagnesii, Agaricus silvaticus, Agaricus silvicola & Portugal & {$[78]$} \\
\hline Spectrophotometry & $\begin{array}{l}\text { Hypholoma fasciculare, Lepista nuda, Lycoperdon molle, Lycoperdon perlatum, Ramaria botrytis } \\
\text { and Tricholoma acerbum }\end{array}$ & Portugal & {$[79]$} \\
\hline Spectrophotometry & $\begin{array}{l}\text { Boletus edulis, Calocybe gambosa, Cantharelus cibarius, Craterellus cornucopioides, Marasmius } \\
\text { oreades }\end{array}$ & Portugal & [94] \\
\hline HPLC-UV & Pleurotus ostreatus & India & {$[81]$} \\
\hline HPLC-UV & Hypsizigus marmoreus & Taiwan & {$[73]$} \\
\hline HPLC-UV & Hypsizigus marmoreus & Taiwan & {$[48]$} \\
\hline
\end{tabular}<smiles>CC1=C(/C=C/C(C)=C/C=C/C(C)=C/C=C/C=C(C)/C=C/C=C(C)/C=C/C2=C(C)CCCC2(C)C)C(C)(C)CCC1</smiles>

$\beta$-Carotene<smiles>CC1=CCCC(C)(C)C1/C=C/C(C)=C/C=C/C(C)=C/C=C/C=C(C)/C=C/C=C(C)/C=C/C1=C(C)CCCC1(C)C</smiles>

$\alpha$-Carotene<smiles>CC/C=C/C(C)=C\C=C\C(C)=C\C=C\C(C)=C\C=C\C=C(C)\C=C\C=C(C)\C=C\C/C(C)=C\CC=C(C)C</smiles><smiles></smiles>

$\beta$-Cryptoxanthin<smiles>CC1=CC(O)CC(C)(C)C1/C=C/C(C)=C/C=C/C(C)=C/C=C/C=C(C)/C=C/C=C(C)/C=C/C1=C(C)CC(O)CC1(C)C</smiles>

Fig. (9). Structures of some major dietary carotenoids.

their characteristic colors and antioxidant properties. Lycopene and $\beta$-carotene are examples of acyclized and cyclized carotenoids, respectively. Due to the presence of the conjugated double bonds, carotenoids can undergo isomerization to cis-trans isomers. Although the trans isomers are more common in foods and are more stable, very little is known about the biological significance of carotenoid isomerization in human health [128]. Carotenoids are thought to be respon- sible for the beneficial properties in preventing human diseases including cardiovascular diseases, cancer and other chronic diseases [129]. They are important dietary sources of vitamin $A$, being $\beta$-Carotene, $\alpha$-carotene, and $\beta$-cryptoxanthin able to function as provitamin A [38].

In recent years their antioxidant properties have been the major focus of research. Carotenoids can react with free radicals and become radicals themselves. They function as a 
chain-breaking antioxidant in a lipid environment, especially under low oxygen partial pressures $[128,130]$. The peroxyl radicals (ROO) formed from lipids (especially polyunsaturated phospholipids) are very damaging to cells. The extensive systems of double bonds make carotenoids susceptible to attack by peroxyl radicals, resulting in the formation of inactive products [122].

$$
\begin{aligned}
\mathrm{RH}+\text { Oxidant initiator } & \longrightarrow \mathrm{R}^{\bullet} \\
\mathrm{R}^{\bullet}+\mathrm{O}_{2} & \longrightarrow \mathrm{ROO} \\
\mathrm{ROO}^{*}+\text { Carotenoids } & \longrightarrow \text { Formation of inactive products }
\end{aligned}
$$

Carotenoids reactivity depends on the length of the chain of conjugated double bonds and the characteristics of the end groups. Carotenoid radicals are stable by virtue of the delocalization of the unpaired electron over the conjugated polyene chain of the molecules. This delocalization also allows addition reactions to occur at many sites on the radical [127].

Overall, wild mushrooms contain different antioxidants such as phenolic compounds, tocopherols, ascorbic acid, and carotenoids which could be extracted for the purpose of being used as functional ingredients namely against chronic diseases related to oxidative stress. Also, mushrooms might be used directly in diet and promote health, taking advantage of the additive and synergistic effects of all the bioactive compounds present.

Public health authorities consider prevention with nutraceuticals as a powerful instrument in maintaining and promoting health, longevity and life quality.

\section{REFERENCES}

[1] Valko, M.; Leibfritz, D.; Moncol, J.; Cronin, M.T.; Mazur, M.; Telser J. Free radicals and antioxidants in normal physiological functions and human disease. Int. J. Biochem. Cell Biol., 2007, 39, 44-84.

[2] Fang, Y.Z.; Yang, S.; Wu, G.Y. Free radicals, antioxidants, and nutrition. Nutrition, 2002, 18, 872-879.

[3] Freidovich, I. Fundamental aspects of reactive oxygen species, or what's the matter with oxygen? Ann. N.Y. Acad. Sci., 1999, 893, 1318.

[4] Machlin, L.J.; Bendich, A. Free radical tissue damage: protective role of antioxidant nutrients. FASEB J., 1987, 1, 441-445.

[5] Fu, S.; Davies, M.J.; Stocker, R.; Dean, R.T. Evidence for roles of radicals in protein oxidation in advanced human atherosclerotic plaque. Biochem. J., 1998, 333, 519-525.

[6] Ridnour, L.A.; Isenberg, J.S.; Espey, M.G.; Thomas, D.D.; Roberts, D.D.; Wink, D.A. Nitric oxide regulates angiogenesis through a functional switch involving thrombospondin-1. Proc. Natl. Acad. Sci. U S A, 2005, 102, 13147-13152.

[7] Valko, M.; Rhodes, C.J.; Moncol, J.; Izakovic, M.; Mazur, M. Free radicals, metals and antioxidants in oxidative stress-induced cancer. Chem. Biol. Interact., 2006, 160, 1-40.

[8] Wei, M.Y.; Cassano, P.A.; Kritchevsky, S.; Harris, T.B.; Holvoet, P.; Crapo, R.O.; Jensen, R.; Newman, A.B.; Bauer, D.C. Oxidized LDL, antioxidants and pulmonary disease. FASEB J., 2004, 18, A908-A908.

[9] Shah, A.M.; Channon, K.M. Free radicals and redox signalling in cardiovascular disease. Heart, 2004, 90, 486-487.

[10] Moreira, P.I.; Santos, M.S.; Oliveira, C.R.; Shenk, J.C.; Nunomura, A.; Smith, M.A.; Zhu, X.; Perry, G. Alzheimer disease and the role of free radicals in the pathogenesis of the disease. CNS Neurol. Disord. Drug Targets, 2008, 7, 3-10.

[11] Barja, G. Free radicals and aging. Trends Neurosci., 2004, 27, 595600 .
[12] Halliwell, B. Antioxidants in human health and disease. Annu. Rev Nutr., 1996, 16, 33-50.

[13] Halliwell, B.; Gutteridge, J.M. Oxygen toxicity, oxygen radicals, transition metals and disease. Biochem. J., 1984, 219, 1-14.

[14] Fries, J.F.; Koop, C.E.; Beadle, C.E.; Cooper, P.P.; England, M.J.; Greaves, R.F.; Sokolov, J.J.; Wright, D. Reducing health care costs by reducing the need and demand for medical services. The Health Project Consortium. N. Engl. J. Med., 1993, 329, 321-325.

[15] Lachance, P.A.; Nakat, Z.; Jeong, W.S. Antioxidants: an integrative approach. Nutrition, 2001, 17, 835-838.

[16] Gutteridge, J.M.; Halliwell, B. Free radicals and antioxidants in the year 2000. A historical look to the future. Ann. N.Y. Acad. Sci., 2000, 899, 136-147.

[17] Halliwell, B.; Gutteridge, J.M.C. Free radicals in biology and medicine (3rd Ed.). Oxford University Press, Oxford, UK, 1999.

[18] Miller, D.M.; Buettner, G.R.; Aust, S.D. Transition metals as catalysts of "autoxidation" reactions. Free Radic. Biol. Med., 1990, 8 , 95-108.

[19] Gilbert, D.L. Fifty years of radical ideas. Ann. N.Y. Acad. Sci., 2000, 899, 1-14.

[20] Cadenas, E.; Sies, H. The lag phase. Free Radic. Res., 1998, 28, 601-609.

[21] Kovacic, P.; Pozos, R.S.; Somanathan, R.; Shangari, N.; O'Brien, P.J. Mechanism of mitochondrial uncouplers, inhibitors, and toxins: focus on electron transfer, free radicals, and structure-activity relationships. Curr. Med. Chem., 2005, 12, 2601-2623.

[22] Halliwell, B.; Gutteridge, J.M. Role of free radicals and catalytic metal ions in human disease: an overview. Methods Enzymol., 1990, 186, 1-85.

[23] Mehrotra, S.; Kakkar, P.; Viswanathan, P.N. Mitochondrial damage by active oxygen species in vitro. Free Radic. Biol. Med., 1991, 10 , 277-285.

[24] Abreu, R.M.V.; Santos, D.J.S.L.; Moreno, A.J.M. Effects of carvedilol and its analog BM-910228 on mitochondrial function and oxidative stress. J. Pharmacol. Exp. Ther., 2000, 295, 1022-1030.

[25] McCord, J.M. The evolution of free radicals and oxidative stress. Am. J. Med., 2000, 108, 652-659.

[26] Ghafourifar, P.; Cadenas, E. Mitochondrial nitric oxide synthase Trends Pharmacol. Sci., 2005, 26, 190-195.

[27] Bergendi, L.; Benes, L.; Durackova, Z.; Ferencik, M. Chemistry, physiology and pathology of free radicals. Life Sci., 1999, 65, 1865-1874

[28] Carr, A.C.; McCall, M.R.; Frei, B. Oxidation of LDL by myeloperoxidase and reactive nitrogen species: reaction pathways and antioxidant protection. Arterioscler. Thromb. Vasc. Biol., 2000, 20, 1716-1723.

[29] Goetz, M.E.; Luch, A. Reactive species: a cell damaging rout assisting to chemical carcinogens. Cancer Lett., 2008, 266, 73-83.

[30] Lee, J.; Koo, N.; Min, D.B. Reactive oxygen species, aging, and antioxidative nutraceuticals. Comp. Rev. Food Sci. Food Safety, 2004, 3, 21-33.

[31] Falandysz, J. Selenium in edible mushrooms. J. Environ. Sci. Health C Environ. Carcinog. Ecotoxicol. Rev., 2008, 26, 256-299.

[32] Johansen, J.S.; Harris, A.K.; Rychly, D.J.; Ergul, A. Oxidative stress and the use of antioxidants in diabetes: linking basic science to clinical practice. Cardiovasc. Diabetol., 2005, 4, 5 .

[33] Pastore, A.; Federici, G.; Bertini, E.; Piemonte, F. Analysis of glutathione: implication in redox and detoxification. Clin. Chim. Acta, 2003, 333, 19-39.

[34] Hensley, K.; Benaksas, E.J.; Bolli, R.; Comp, P.; Grammas, P.; Hamdheydari, L.; Mou, S.; Pye, Q.N.; Stoddard, M.F.; Wallis, G.; Williamson, K.S.; West, M.; Wechter, W.J.; Floyd, R.A. New perspectives on vitamin E: gamma-tocopherol and carboxyelthylhydroxychroman metabolites in biology and medicine. Free Radic. Biol. Med., 2004, 36, 1-15.

[35] Ames, B.N. Dietary carcinogens and anticarcinogens. Oxygen radicals and degenerative diseases. Science, 1983, 221, 1256-1264.

[36] Branen, A.L. Toxicology and biochemistry of butylated hydroxyanisole and butylated hydroxytoluene. J. Am. Oil Chem. Soc., 1975, 52, 59-63.

[37] Ramarathnam, N.; Osawa, T.; Ochi, H.; Kawakishi, S. The contribution of plant food antioxidants to human health. Trends Food Sci. Tech., 1995, 6, 75-82.

[38] Liu, R.H. Potential synergy of phytochemicals in cancer prevention: mechanism of action. J. Nutr., 2004, 134, 3479S-3485S. 
[39] Soobrattee, M.A.; Neergheen, V.S.; Luximon-Ramma, A.; Aruoma, O.I.; Bahorun, T. Phenolics as potential antioxidant therapeutic agents: Mechanism and actions. Mutation ResearchFundamental and Molecular Mechanisms of Mutagenesis, 2005, 579, 200-213.

[40] Temple, N.J. Antioxidants and disease: More questions than answers. Nutr. Res., 2000, 20, 449-459.

[41] Willett, W.C. Diet and Health - What should we eat. Science, 1994, 264, 532-537.

[42] Willett, W.C. Balancing life-style and genomics research for disease prevention. Science, 2002, 296, 695-698.

[43] Liu, R.H. Health benefits of fruit and vegetables are from additive and synergistic combinations of phytochemicals. Am. J. Clin. Nutr., 2003, 78, 517S-520S.

[44] Chang, R. Functional properties of edible mushrooms. Nutr. Rev., 1996, 54, S91-93.

[45] Chang, S. Global impact of edible and medicinal mushrooms on human welfare in the 21st century: Nongreen revolution. Int. J. Med. Mushrooms, 1999, 1, 1-7.

[46] Lindequist, U.; Niedermeyer, T.H.J.; Julich, W.D. The pharmacological potential of mushrooms. eCAM, 2005, 2, 285-299.

[47] Wasser, S.P.; L., W.A. Medicinal properties of substances occurring in higher Basidiomycete mushrooms: current perspective (review). Int. J. Med. Mushrooms, 1999, 1, 31-62.

[48] Lee, Y.-L.; Jian, S.-Y.; Lian, P.-Y.; Mau, J.-L. Antioxidant properties of extracts from a white mutant of the mushroom Hypsizigus marmoreus. J. Food Comp. Anal., 2008, 21, 116-124.

[49] Mau, J.L.; Lin, H.C.; Chen, C.C. Antioxidant properties of several medicinal mushrooms. J. Agric. Food Chem., 2002, 50, 6072-6077.

[50] Mau, J.-L.; Lin, H.-C.; Song, S.-F. Antioxidant properties of several specialty mushrooms. Food Res. Int., 2002, 35, 519-526.

[51] Murcia, M.A.; Martinez-Tome, M.; Jimenez, A.M.; Vera, A.M.; Honrubia, M.; Parras, P. J. Antioxidant activity of edible fungi (truffles and mushrooms): losses during industrial processing. Food Prot., 2002, 65, 1614-1622.

[52] Yang, J.-H.; Lin, H.-C.; Mau, J.-L. Antioxidant properties of several commercial mushrooms. Food Chem., 2002, 77, 229-235.

[53] Cheung, L.M.; Cheung, P.C.K.; Ooi, V.E.C. Antioxidant activity and total phenolics of edible mushroom extracts. Food Chem., 2003, $81,249-255$.

[54] Lee, B.C.; Baea, J.T.; Pyoa, H.B.; Choeb, T.B.; Kimc, S.W.; Hwangc, H.J.; Yun, J.W. Biological activities of the polysaccharides produced from submerged culture of the edible Basidiomycete Grifola frondosa. Enzyme Microb. Technol., 2003, 32, 574581.

[55] Song, Y.S.; Kim, S.H.; Sa, J.H.; Jin, C.; Lim, C.J.; Park, E.H. Antiangiogenic, antioxidant and xanthine oxidase inhibition activities of the mushroom Phellinus linteus. J. Ethnopharmacol., 2003, 88, $113-116$.

[56] Acharya, K.; Samui, K.; Rai, M.; Dutta, B.B.; Acharya, R. Antioxidant and nitric oxide synthase activation properties of Auricularia auricula. Indian J. Exp. Biol., 2004, 42, 538-540.

[57] Mau, J.-L.; Chang, C.-N.; Huang, S.-J.; Chen, C.-C. Antioxidant properties of methanolic extracts from Grifola frondosa, Morchella esculenta and Termitomyces albuminosus mycelia. Food Chem., 2004, 87, 111-118.

[58] Acharya, K.; Yonzone, P.; Rai, M.; Rupa, A. Antioxidant and nitric oxide synthase activation properties of Ganoderma applanatum. Indian J. Exp. Biol., 2005, 43, 926-929.

[59] Cheung, L.M.; Cheung, P.C.K. Mushroom extracts with antioxidant activity against lipid peroxidation. Food Chem., 2005, 89, 403-409.

[60] Cui, Y.; Kim, D.S.; Park, K.C. Antioxidant effect of Inonotus obliquus. J. Ethnopharmacol., 2005, 96, 79-85.

[61] Lo, K.M.; Cheung, P.C.K. Antioxidant activity of extracts from the fruiting bodies of Agrocybe aegerita var. alba. Food Chem., 2005, 89, 533-539.

[62] Choi, Y.; Lee, S.M.; Chun, J.; Lee, H.B.; Lee, J. Influence of heat treatment on the antioxidant activities and polyphenolic compounds of Shiitake (Lentinus edodes) mushroom. Food Chem., 2006, 99, 381-387.

[63] Elmastas, M.; Turkekul, I.; Ozturk, L.; Gulcin, I.; Isildak, O.; Aboul-Enein, H.Y. Antioxidant activity of two wild edible mushrooms (Morchella vulgaris and Morchella esculanta) from North Turkey. Comb. Chem. High Throughput Screen, 2006, 9, 443-448.
[64] Puttaraju, N.G.; Venkateshaiah, S.U.; Dharmesh, S.M.; Urs, S.M.; Somasundaram, R. Antioxidant activity of indigenous edible mushrooms. J. Agric. Food Chem., 2006, 54, 9764-9772.

[65] Ribeiro, B.; Rangel, J.; Valentao, P.; Baptista, P.; Seabra, R.M.; Andrade, P.B. Contents of carboxylic acids and two phenolics and antioxidant activity of dried portuguese wild edible mushrooms. $J$. Agric. Food Chem., 2006, 54, 8530-8537.

[66] Hu, S.H.; Liang, Z.C.; Chia, Y.C.; Lien, J.L.; Chen, K.S.; Lee, M.Y.; Wang, J.C. Antihyperlipidemic and antioxidant effects of extracts from Pleurotus citrinopileatus. J. Agric. Food Chem., 2006 , 54, 2103-2110.

[67] Barros, L.; Ferreira, M.-J.; Queirós, B.; Ferreira, I.C.F.R.; Baptista, P. Total phenols, ascorbic acid, $\beta$-carotene and lycopene in Portuguese wild edible mushrooms and their antioxidant activities. Food Chem., 2007, 103, 413-419.

[68] Barros, L.; Baptista, P.; Ferreira, I.C.F.R. Effect of Lactarius piperatus fruiting body maturity stage on antioxidant activity measured by several biochemical assays. Food Chem. Toxicol., 2007, 45, 1731-1737.

[69] Dore, C.M.P.G.; Azevedo, T.C.G.; de Souza, M.C.R.; Rego, L.A.; de Dantas, J.C.M.; Silva, F.R.F.; Rocha, H.A.O.; Basela, I.G.; Leite, E.L. Antiinflammatory, antioxidant and cytotoxic actions of beta-glucan-rich extract from Geastrum saecatum mushroom. Int. Immunopharmacol., 2007, 7, 1160-1169.

[70] Elmastas, M.; Isildak, O.; Turkekul, I.; Temur, N. Determination of antioxidant activity and antioxidant compounds in wild edible mushrooms. J. Food Comp. Anal., 2007, 20, 337-345.

[71] Ferreira, I.C.F.R.; Baptista, P.; Vilas-Boas, M.; Barros, L. Freeradical scavenging capacity and reducing power of wild edible mushrooms from northeast Portugal: Individual cap and stipe activity. Food Chem., 2007, 100, 1511-1516.

[72] Kitzberger, C.S.G.; Smania, A.; Pedrosa, R.C.; Ferreira, S.R.S. Antioxidant and antimicrobial activities of shiitake (Lentinula edodes) extracts obtained by organic solvents and supercritical fluids. J. Food Eng., 2007, 80, 631-638.

[73] Lee, I.K.; Kim, Y.S.; Jang, Y.W.; Jung, J.Y.; Yun, B.S. Antioxidant properties of various extracts from Hypsizigus marmoreus. Food Chem., 2007, 104, 1-9.

[74] Ng, L.T.; Wu, S.J.; Tsai, J.Y.; Lai, M.N. Antioxidant activities of cultured Armillariella mellea. Prikl Biokhim Mikrobiol., 2007, 43, 495-500.

[75] Oliveira, O.M.; Vellosa, J.C.; Fernandes, A.S.; Buffa-Filho, W.; Hakime-Silva, R.A.; Furlan, M.; Brunetti, I.L. Antioxidant activity of Agaricus blazei. Fitoterapia, 2007, 78, 263-264.

[76] Tsai, S. Y.; Tsai, H.L.; Mau, J.L. Antioxidant properties of Agaricus blazei, Agrocybe cylindracea, and Boletus edulis. Lwt-Food Sci. Technol., 2007, 40, 1392-1402.

[77] Turkoglu, A.; Duru, M.E.; Mercan, N.; Kivrak, I.; Gezer, K. Antioxidant and antimicrobial activities of Laetiporus sulphureus (Bull.) Murrill. Food Chem., 2007, 101, 267-273.

[78] Barros, L.; Falcão, S.; Baptista, P.; Freire, C.; Vilas-Boas, M.; Ferreira, I.C.F.R. Antioxidant activity of Agaricus sp. mushrooms by chemical, biochemical and electrochemical assays. Food Chem., 2008, 111, 61-66.

[79] Barros, L.; Venturini, B.A.; Baptista, P.; Estevinho, L.M.; Ferreira, I.C.F.R. Chemical Composition and Biological Properties of Portuguese Wild Mushrooms: A comprehensive study. J. Agric. Food Chem., 2008, 56, 3856-3862.

[80] Barros, L.; Baptista, P.; Correia, D.M.; Morais, J.S.; Ferreira, I.CF.R. Effects of conservation treatment and cooking on the chemical composition and antioxidant activity of Portuguese wild edible mushrooms. J. Agric. Food Chem., 2007, 55, 4781-4788.

[81] Jayakumar, T.; Thomas, P.A.; Geraldine, P. In-vitro antioxidant activities of an ethanolic extract of the oyster mushroom, Pleurotus ostreatus. Innovat. Food Sci. Emerg. Technol., 2008, In Press, Corrected Proof.

[82] Kim, M.Y.; Seguin, P.; Ahn, J.K.; Kim, J.J.; Chun, S.C.; Kim, E.H.; Seo, S.H.; Kang, E.Y.; Kim, S.L.; Park, Y.J.; Ro, H.M.; Chung, I.M. Phenolic compound concentration and antioxidant activities of edible and medicinal mushrooms from Korea. J. Agric. Food Chem., 2008, 56, 7265-7270.

[83] Sarikurkcu, C.; Tepe, B.; Yamac, M. Evaluation of the antioxidant activity of four edible mushrooms from the Central Anatolia. Bioresour. Technol., 2008, 99, 6651-6655.

[84] Soares, A.A.; de Souza, C.G.M.; Daniel, F.M.; Ferrari, G.P.; da Costa, S.M.G.; Peralta, R.M. Antioxidant activity and total pheno- 
lic content of Agaricus brasiliensis (Agaricus blazei Murril) in two stages of maturity. Food Chem., 2009, 112, 775-781.

[85] Valentão, P.; Andrade, P.B.; Rangel, J.; Ribeiro, B.; Silva, B.M.; Baptista, P.; Seabra, R.M. Effect of the conservation procedure on the contents of phenolic compounds and organic acids in Chanterelle (Cantharellus cibarius) mushroom. J. Agric. Food Chem., 2005, 53, 4925-4931.

[86] Barros, L.; Baptista, P.; Estevinho, L.M.; Ferreira, I.C.F.R. Effect of fruiting body maturity stage on chemical composition and antimicrobial activity of Lactarius sp. mushrooms. J. Agric. Food Chem., 2007, 55, 8766-8771.

[87] Barros, L.; Correia, D.M.; Ferreira, I.C.F.R.; Baptista, P.; SantosBuelga, C. Optimization of the determination of tocopherols in Agaricus sp. edible mushrooms by a normal phase liquid chromatographic method. Food Chem., 2008, 110, 1046-1050.

[88] Barros, L.; Dueñas, M.; Ferreira, I.C.F.R.; Baptista, P.; SantosBuelga, C. Phenolic acids determination by HPLC-DAD-ESI/MS in sixteen different Portuguese wild mushrooms species. Food Chem. Toxicol., 2008, (In Press).

[89] Mattila, P.; Konko, K.; Eurola, M.; Pihlava, J.M.; Astola, J.; Vahteristo, L.; Hietaniemi, V.; Kumpulainen, J.; Valtonen, M.; Piironen, V. Contents of vitamins, mineral elements, and some phenolic compounds in cultivated mushrooms. J. Agric. Food Chem., 2001, 49, 2343-2348.

[90] Jaworska, G.; Bernas, E.; Cichon, Z.; Possinger, P. Establishing the optimal period of storage for frozen Agaricus bisporus, depending on the preliminary processing applied. Int. J. Refrigeration, $\mathbf{2 0 0 8}$, 31, 1042-1050.

[91] Valentao, P.; Lopes, G.; Valente, M.; Barbosa, P.; Andrade, P.B.; Silva, B.M.; Baptista, P.; Seabra, R.M. Quantitation of nine organic acids in wild mushrooms. J. Agric. Food Chem., 2005, 53, 36263630.

[92] Ribeiro, B.; Valentao, P.; Baptista, P.; Seabra, R.M.; Andrade, P.B. Phenolic compounds, organic acids profiles and antioxidative properties of beefsteak fungus (Fistulina hepatica). Food Chem. Toxicol., 2007, 45, 1805-1813.

[93] Mau, J.L.; Chao, G.R.; Wu, K.T. Antioxidant properties of methanolic extracts from several ear mushrooms. J. Agric. Food Chem., 2001, 49, 5461-5467.

[94] Barros, L.; Cruz, T.; Baptista, P.; Estevinho, L.M.; Ferreira, I.C.F.R. Wild and commercial mushrooms as source of nutrients and nutraceuticals. Food Chem. Toxicol., 2008, 46, 2742-2747.

[95] Agrahar-Murugkar, D.; Subbulakshmi, G. Nutritional value of edible wild mushrooms collected from the Khasi hills of Meghalaya. Food Chem., 2005, 89, 599-603.

[96] George, S.; Brat, P.; Alter, P.; Amiot, M.J. Rapid determination of polyphenols and vitamin $\mathrm{C}$ in plant-derived products. J. Agric. Food Chem., 2005, 53, 1370-1373.

[97] Apak, R.; Guclu, K.; Demirata, B.; Ozyurek, M.; Celik, S.E.; Bektasoglu, B.; Berker, K.I.; Ozyurt, D. Comparative evaluation of various total antioxidant capacity assays applied to phenolic compounds with the CUPRAC assay. Molecules, 2007, 12, 1496-1547.

[98] Bravo, L. Polyphenols: chemistry, dietary sources, metabolism, and nutritional significance. Nutr. Rev., 1998, 56, 317-333.

[99] Hall, C. Sources of natural antioxidants: oilseeds, nuts, cereals, legumes, animal products and microbial sources. In Antioxidants in food: practical applications. Pokorný, J.; Yanishlieva, N.; Gordon, M., Eds.; Woodhead Publishing Limited: Cambridge England, 2001, pp 159-209.

[100] Wright, J.S.; Johnson, E.R.; DiLabio, G.A. Predicting the activity of phenolic antioxidants: theoretical method, analysis of substituent effects, and application to major families of antioxidants. $J$. Am. Chem. Soc., 2001, 123, 1173-1183.

[101] Baum, B.; Perun, A.L. Antioxidant efficiency versus structure. Spe Transactions, 1962, 2, 250-259.

[102] Cuvelier, M.E.; Richard, H.; Berset, C. Comparison of the antioxidative activity of some acid-phenols - structure activity relationship. Biosci. Biotechnol. Biochem., 1992, 56, 324-325.

[103] Iwashina, T. The structure and distribution of the flavonoids in plants. J. Plant Res., 2000, 113, 287-299.

[104] Nijveldt, R.J.; van Nood, E.; van Hoorn, D.E.C.; Boelens, P.G.; van Norren, K.; van Leeuwen, P.A.M. Flavonoids: a review of probable mechanisms of action and potential applications. Am. J. Clin. Nutr., 2001, 74, 418-425.

[105] Hollman, P.C.H.; Arts, I.C.W. Flavonols, flavones and flavanols nature, occurrence and dietary burden. J. Sci. Food Agric., 2000, 80, 1081-1093.

[106] Le Marchand, L. Cancer preventive effects of flavonoids--a review. Biomed. Pharmacother., 2002, 56, 296-301.

[107] Macheix, J.; Fleuriet, A. Flavonoids in health and disease. Marcel Dekker Inc, New York 1998.

[108] Kamal-Eldin, A.; Appelqvist, L.A. The chemistry and antioxidant properties of tocopherols and tocotrienols. Lipids, 1996, 31, 671701.

[109] Blokhina, O.; Virolainen, E.; Fagerstedt, K.V. Antioxidants, oxidative damage and oxygen deprivation stress: a review. Ann. Bot (Lond), 2003, 91 Spec No, 179-194.

[110] Burton, G.W.; Traber, M.G. Vitamin E: antioxidant activity, biokinetics, and bioavailability. Annu. Rev. Nutr., 1990, 10, 357-382

[111] Lampi, A.M.; Kataja, L.; Kamal-Eldin, A.; Vieno, P. Antioxidant activities of alpha- and gamma-tocopherols in the oxidation of rapeseed oil triacylglycerols. J. Am. Oil Chem. Soc. 1999, 76, 749755.

[112] Schwenke, D.C. Does lack of tocopherols and tocotrienols put women at increased risk of breast cancer? J. Nutr. Biochem., 2002, $13,2-20$

[113] Traber, M.G. Vitamin E. In Modern Nutrition in Health and Disease. Shils, M.E.; Olson, J.A.; Shike, M.; Ross, A.C., Eds.; Baltimore: Williams and Wilkins (10th ed.), 1999, pp 347-362.

[114] Davey, M.W.; Van Montagu, M.; Inze, D.; Sanmartin, M.; Kanellis, A.; Smirnoff, N.; Benzie, I.J.J.; Strain, J.J.; Favell, D.; Fletcher, J. Plant L-ascorbic acid: chemistry, function, metabolism, bioavailability and effects of processing. J. Sci. Food Agric., 2000, 80, 825860.

[115] Giovannoni, J.J. Completing a pathway to plant vitamin C synthesis. Proc. Nat. Acad. Sci. USA, 2007, 104, 9109-9110.

[116] Hancock, R.D.; Viola, R. Biosynthesis and catabolism of Lascorbic acid in plants. Crit. Rev. Plant Sci., 2005, 24, 167-188.

[117] Klein, B.P.; Perry, A.K. Ascorbic-acid and vitamin-A activity in selected vegetables from different geographical areas of the UnitedStates. J. Food Sci., 1982, 47, 941-945.

[118] Sies, H.; Stahl, W.; Sundquist, A.R. Antioxidant functions of vitamins - vitamin-E and vitamin-C, beta-Carotene, and other carotenoids. Ann. N.Y. Acad. Sci., 1992, 669, 7-20.

[119] Li, Y.; Schellhorn, H.E. New developments and novel therapeutic perspectives for vitamin C. J. Nutr., 2007, 137, 2171-2184.

[120] Burton, G.W.; Ingold, K.U. Vitamin-E - Application of the principles of physical organic-chemistry to the exploration of its structure and function. Acc. Chem. Res., 1986, 19, 194-201.

[121] Ingold, K.U.; Bowry, V.W.; Stocker, R.; Walling, C. Autoxidation of lipids and antioxidation by alpha-tocopherol and ubiquinol in homogeneous solution and in aqueous dispersions of lipids - unrecognized consequences of lipid particle-size as exemplified by oxidation of human low-density-lipoprotein. Proc. Nat. Acad. Sci. USA, 1993, 90, 45-49.

[122] Chew, B.P. Antioxidant vitamins affect food animal immunity and health. J. Nutr., 1995, 125, S1804-S1808.

[123] Nagaoka, S.; Kakiuchi, T.; Ohara, K.; Mukai, K. Kinetics of the reaction by vitamin $\mathrm{E}$ is regenerated which natural by vitamin $\mathrm{C}$. Chem. Phys. Lipids, 2007, 146, 26-32.

[124] Johnson, E.J. The role of carotenoids in human health. Nutr. Clin. Care, 2002, 5, 56-65.

[125] Mangels, A.R.; Holden, J.M.; Beecher, G.R.; Forman, M.R.; Lanza, E. Carotenoid content of fruits and vegetables - an evaluation of analytic data. J. Am. Diet. Assoc., 1993, 93, 284-296.

[126] Gerster, H. The potential role of lycopene for human health. J. Am. Coll. Nutr., 1997, 16, 109-126.

[127] Britton, G. Structure and properties of carotenoids in relation to function. FASEB J., 1995, 9, 1551-1558.

[128] Rao, A.V.; Rao, L.G. Carotenoids and human health. Pharmacol. Res., 2007, 55, 207-216.

[129] Paiva, S.A.; Russell, R.M. Beta-carotene and other carotenoids as antioxidants. J. Am. Coll. Nutr., 1999, 18, 426-433.

[130] Burton, G.W.; Ingold, K.U. beta-Carotene: an unusual type of lipid antioxidant. Science, 1984, 224, 569-573. 